\title{
LARGE DEVIATION ESTIMATES OF THE CROSSING PROBABILITY FOR PINNED GAUSSIAN PROCESSES
}

\author{
LUCIA CARAMELLINO*** AND \\ BARBARA PACCHIAROTTI, ${ }^{* * *}$ Università di Roma Tor Vergata
}

\begin{abstract}
The paper deals with the asymptotic behavior of the bridge of a Gaussian process conditioned to stay in $n$ fixed points at $n$ fixed past instants. In particular, functional large deviation results are stated for small time. Several examples are considered: integrated or not fractional Brownian motions and $m$-fold integrated Brownian motion. As an application, the asymptotic behavior of the exit probability is studied and used for the practical purpose of the numerical computation, via Monte Carlo methods, of the hitting probability up to a given time of the unpinned process.
\end{abstract}

Keywords: Conditioned Gaussian process; reproducing kernel Hilbert spaces; large deviations; exit time probability; Monte Carlo method

2000 Mathematics Subject Classification: Primary 60F10; 60G15

Secondary $65 \mathrm{C} 05$

\section{Introduction}

Simple formulae for the crossing probability in small time for pinned processes have been recently investigated in the literature, because of their use in improving the performance of the numerical simulation of processes to be killed when a prescribed boundary is reached. The idea underlying the application is simple. In fact, consider the generic step of the simulation procedure: we have generated the process of interest, say $X$, at some $n \geq 1$ fixed instants $0<T_{1}<\cdots<T_{n}$, observing the positions $X_{T_{1}}=x_{1}, \ldots, X_{T_{n}}=x_{n}$. In order to obtain the exit time, we simulate the process again, at time $T_{n}+\varepsilon$, and if the observed position $X_{T_{n}+\varepsilon}=y$ reaches the boundary then the crossing is achieved. This gives rise to an overestimate of the exit time, which can incur a significant error, as observed by many authors. One way to overcome this difficulty is to compute the crossing probability of the pinned process, that is, for the conditional process $\left(X_{T_{n}+\varepsilon t}\right)_{0 \leq t \leq 1}$ given all the past observations $X_{T_{1}}=x_{1}, \ldots, X_{T_{n}}=x_{n}$ and the present observation $X_{T_{n}+\varepsilon}=y$, and to use it in order to decide if the boundary has been breached or not. Let us stress that in the general case, no closed formulae are available, so that such a procedure is carried out with an approximation (by large deviations as $\varepsilon \rightarrow 0$ ) of the exit probability.

In the case of diffusion processes the Markov property allows us to work with the bridge process between the observations at times $T_{n}$ and $T_{n}+\varepsilon$. This case has been widely studied

Received 2 November 2007; revision received 31 March 2008.

* Postal address: Dipartimento di Matematica, Università di Roma Tor Vergata, Via della Ricerca Scientifica, I-00133

Roma, Italy.

** Email address: caramell@mat.uniroma2.it

*** Email address: pacchiar@mat.uniroma2.it 
in the literature; see, e.g. [2] and the references therein. This approach obviously fails if a non-Markovian process is studied, in which case we have to consider all the past observations and to handle the bridge of the conditional process.

The present paper deals with the large deviation asymptotic behavior of the exit probability of such a pinned process whenever the original process is a continuous Gaussian process (and, in particular, not necessarily a Markovian process). Our wide class of examples can be split into two main sets.

First, we consider the fractional Brownian motion, which is widely used in risk theory modeling (see, e.g. [3]). As a consequence, we can handle the semimartingale process resulting from a linear combination between a fractional Brownian motion with Hurst index greater than $\frac{3}{4}$ and a standard Brownian motion, independent of each other, a process providing a promising tool to set up a non-Markovian model in mathematical finance (see [6]).

Secondly, we can deal with an integrated Gaussian process, that is, a process defined as the integral with respect to the Lebesgue measure of a Gaussian process. As an example, we obtain the integrated fractional Brownian motion, which is linked to fractal properties of solutions to the inviscid Burgers equation. Note that the law of its maximum, analyzed for example in [13], is strictly connected to level crossing probabilities. Furthermore, we can consider the $m$-fold iterated Brownian motion (see, e.g. [5]) and, in particular, the integrated Brownian motion, which has interesting applications in nonparametric estimating in statistics (see, e.g. [11] and the references therein) and is used in metrology as a model for the atomic clock error, whose precision and re-synchronization are strictly related to the level crossing (see, e.g. [9]).

The paper is organized as follows. After a brief recall of some well-known results related to large deviations for Gaussian processes (Section 2), we first obtain a functional large deviation result, for small time, for Gaussian processes conditioned to stay in $n$ fixed positions $x_{1}, \ldots, x_{n}$ at $n$ fixed instants $T_{1}<\cdots<T_{n}$ (Section 3). In Section 4 we state a functional large deviation principle for the bridge of such conditional processes. Let us stress that, surprisingly, we obtain a degenerate kind of large deviations for Gaussian processes having a quite smooth covariance function (e.g. for integrated Gaussian processes), and so we give some refined results which also allow us to handle these cases. In particular, we obtain examples of an interesting and nontrivial asymptotic behavior, in which the (nondegenerate) large deviation speed is different according to the conditional process or its bridge: the speed associated to the bridge can be much faster than the one associated to the conditional process. Finally, in Section 5 we give the asymptotic behavior, in terms of large deviations, of the probability of crossing one or two possibly time-dependent levels, and we propose some numerical results concerning the fractional Brownian motion.

\section{Large deviations for Gaussian processes}

We briefly recall here some main facts we are going to use related to the large deviation theory for Gaussian processes. There are many references in the literature on this topic, where all details and proofs may be found; some classical references include [1], [7], and [8]. Without loss of generality, we can consider centered Gaussian processes.

Throughout the paper, $C([0,1])$ will denote the set of the continuous paths on $[0,1]$, endowed with the topology induced by the sup-norm. Moreover, $\mathcal{M}[0,1]$ will be its dual, i.e. the set of signed Borel measures on $[0,1]$, and, for any $\lambda \in \mathcal{M}[0,1],\langle\lambda, h\rangle=\int_{0}^{1} h_{t} \lambda(\mathrm{d} t), h \in C([0,1])$, where $\langle\lambda, \cdot\rangle$ will stand for the associated linear functional. 
A continuous process $U=\left(U_{t}\right)_{t \in[0,1]}$, defined on some probability space $(\Omega, \mathcal{F}, \mathrm{P})$, is a centered Gaussian process if, for any $\lambda \in \mathcal{M}[0,1],\langle\lambda, U\rangle=\int_{0}^{1} U_{t} \lambda(\mathrm{d} t)$ is a centered Gaussian random variable $(\mathrm{RV})$ taking values on $\mathbb{R}$. The associated continuous covariance function $k(t, s)=\operatorname{cov}\left(U_{t}, U_{s}\right), t, s \in[0,1]$, plays a crucial role. For example, we have

$$
\operatorname{var}(\langle\lambda, U\rangle)=\int_{0}^{1} \int_{0}^{1} k(t, s) \lambda(\mathrm{d} t) \lambda(\mathrm{d} s) \quad \text { for any } \lambda \in \mathcal{M}[0,1] .
$$

In addition to $k$, another important instrument for handling Gaussian processes is the associated reproducing kernel Hilbert space, $\mathscr{H}$. It is a Hilbert space in $C([0,1])$, which is usually defined through the following (dense with respect to a suitable norm) subset:

$$
\mathcal{L}=\left\{h \in C([0,1]): h_{t}=\int_{0}^{1} k(t, s) \lambda(\mathrm{d} s) \text { with } \lambda \in \mathcal{M}[0,1]\right\} .
$$

Let us be a little more precise about $\mathscr{H}$. First, let $\mu$ denote the measure induced by the Gaussian process $U: \mu(A)=\mathrm{P}(U \in A)$ for any Borel set $A$ in $C([0,1])$. Let $\Gamma \subset L^{2}(\mu)$ be defined as the following set of (real) Gaussian RVs:

$$
\Gamma=\{Y: Y(\cdot)=\langle\lambda, \cdot\rangle \text { with } \lambda \in \mathcal{M}[0,1]\}
$$

It immediately follows that, for $Y_{1}, Y_{2} \in \Gamma$ with $Y_{i}(\cdot)=\left\langle\lambda_{i}, \cdot\right\rangle, i=1,2$,

$$
\operatorname{cov}\left(Y_{1}, Y_{2}\right)=\left(Y_{1}, Y_{2}\right)_{L^{2}(\mu)}=\int_{0}^{1} \int_{0}^{1} k(t, s) \lambda_{1}(\mathrm{~d} t) \lambda_{2}(\mathrm{~d} s),
$$

where, from now on, ' $(\cdot, \cdot)$ ' denotes an inner product. We now define

$$
H=\bar{\Gamma}^{\|\cdot\|_{L^{2}(\mu)}} .
$$

Obviously, $H$ is a closed subspace of $L^{2}(\mu)$ and is indeed a set of Gaussian RVs taking values on $\mathbb{R}$. Moreover, it becomes a Hilbert space if endowed with the inner product

$$
\left(Y_{1}, Y_{2}\right)_{H}=\left(Y_{1}, Y_{2}\right)_{L^{2}(\mu)}, \quad Y_{1}, Y_{2} \in H
$$

We now set the following map:

$$
\begin{aligned}
\&: H & \rightarrow C([0,1]), \\
Y & \mapsto(\& Y)_{t}=\int x_{t} Y(x) \mu(\mathrm{d} x) \equiv \mathrm{E}\left(U_{t} Y\right) .
\end{aligned}
$$

It can be shown that $\delta$ is a linear, one-to-one, and continuous map, so that $\delta^{-1}: \& H \rightarrow H$ is a well-posed continuous and linear map. The reproducing kernel Hilbert space $\mathscr{H}$ is defined as the image of $H$ through 8 , i.e.

$$
\mathscr{H}=s H \equiv\left\{h \in C([0,1]): h_{t}=(S Y)_{t}, Y \in H\right\} .
$$


Finally, setting

$$
\left(h_{1}, h_{2}\right)_{\mathscr{H}}=\left(\Im^{-1} h_{1}, \varsigma^{-1} h_{2}\right)_{H} \equiv\left(\Im^{-1} h_{1}, \varsigma^{-1} h_{2}\right)_{L^{2}(\mu)}, \quad h_{1}, h_{2} \in \mathscr{H},
$$

then $(\cdot, \cdot)_{\mathscr{H}}$ is an inner product on $\mathscr{H}$, which in turn makes $\mathscr{H}$ a Hilbert space. This is the rigorous definition of the reproducing kernel Hilbert space associated to a (centered) Gaussian process. Finally, it immediately follows that

$$
\mathscr{H}=\overline{\mathcal{L}}^{\|\cdot\|_{\mathscr{H}}} \quad \text { with } \mathcal{L}=\left\{x \in C([0,1]): x_{t}=\int_{0}^{1} k(t, s) \lambda(\mathrm{d} s) \text { with } \lambda \in \mathcal{M}[0,1]\right\} .
$$

In the sequel we will speak about 'the reproducing kernel Hilbert space associated to the covariance function $k(t, s)^{\prime}$. In fact, given a continuous, symmetric, and positive definite function $k(t, s)$ defined on $[0,1] \times[0,1]$, we can build a centered and continuous Gaussian process $U=\left(U_{t}\right)_{t \in[0,1]}$ having $k$ as its covariance function. Now, the associated reproducing kernel Hilbert space is naturally defined.

The main property we are going to use is related to the Cramèr transform (see, e.g. [8]).

Theorem 2.1. (Cramèr transform.) Let I denote the Cramèr transform, that is,

$$
I(x)=\sup _{\lambda \in \mathcal{M}[0,1]}\left(\langle\lambda, x\rangle-\log \mathrm{E}\left(\mathrm{e}^{\langle\lambda, U\rangle}\right)\right)=\sup _{\lambda \in \mathcal{M}[0,1]}\left(\langle\lambda, x\rangle-\frac{1}{2} \int_{0}^{1} \int_{0}^{1} k(t, s) \lambda(\mathrm{d} t) \lambda(\mathrm{d} s)\right) .
$$

Then,

$$
I(x)= \begin{cases}\frac{1}{2}\|x\|_{\mathcal{H}}^{2} & \text { if } x \in \mathcal{H} \\ +\infty & \text { otherwise }\end{cases}
$$

Now suppose having a family of continuous Gaussian processes $\left\{U^{\varepsilon}\right\}_{\varepsilon}$; is it possible to determine a large deviation principle? Because of the special form of the Laplace transform for Gaussian measures, a large deviation principle can be stated if a suitable asymptotic behavior holds for the Laplace transforms, as summarized in the following theorem.

Theorem 2.2. Let $\left\{U^{\varepsilon}\right\}_{\varepsilon}$ be a family of continuous Gaussian processes. Let $\gamma_{\varepsilon}$ be an infinitesimal function, i.e. $\lim _{\varepsilon \rightarrow 0} \gamma_{\varepsilon}=0$, and suppose that, for any $\lambda \in \mathcal{M}[0,1]$,

$$
0=\lim _{\varepsilon \rightarrow 0} \mathrm{E}\left(\left\langle\lambda, U^{\varepsilon}\right\rangle\right) \text { and } \Lambda(\lambda)=\lim _{\varepsilon \rightarrow 0} \frac{\operatorname{var}\left(\left\langle\lambda, U^{\varepsilon}\right\rangle\right)}{\gamma_{\varepsilon}^{2}} \equiv \int_{0}^{1} \int_{0}^{1} \bar{k}(t, s) \lambda(\mathrm{d} t) \lambda(\mathrm{d} s)
$$

for some continuous, symmetric, and positive definite function $\bar{k}$. Then, $\left\{U^{\varepsilon}\right\}_{\varepsilon}$ satisfies a large deviation principle on $C([0,1])$, with inverse speed $\gamma_{\varepsilon}^{2}$ and (good) rate function

$$
I(h)= \begin{cases}\frac{1}{2}\|h\|_{\overline{\mathscr{H}}}^{2} & \text { if } h \in \overline{\mathscr{H}} \\ +\infty & \text { otherwise }\end{cases}
$$

where $\overline{\mathscr{H}}$ and $\|\cdot\|_{\overline{\mathscr{H}}}$ respectively denote the reproducing kernel Hilbert space and the related norm associated to the covariance function $\bar{k}$. 
Let us recall, once and for all, that the sentence ' $\left\{U^{\varepsilon}\right\}_{\varepsilon}$ satisfies a large deviation principle on $C([0,1])$ with inverse speed $\gamma_{\varepsilon}^{2}$ and (good) rate function $I$ ' means that $\lim _{\varepsilon \rightarrow 0} \gamma_{\varepsilon}=0$, the set $\{I \leq a\}$ is compact in $C([0,1])$ for any fixed $a$, and that the following inequalities hold:

- for any open set $G$ in $C([0,1]), \liminf _{\varepsilon \rightarrow 0} \gamma_{\varepsilon}^{2} \log \mathrm{P}\left(U^{\varepsilon} \in G\right) \geq-\inf _{h \in G} I(h)$;

- for any closed set $F$ in $C([0,1]), \limsup _{\varepsilon \rightarrow 0} \gamma_{\varepsilon}^{2} \log \mathrm{P}\left(U^{\varepsilon} \in F\right) \leq-\inf _{h \in F} I(h)$.

For the sake of convenience, Theorem 2.2 is written for a noncentered family of Gaussian processes, even if it requires that the expected path weakly converges to 0 . The idea of the proof of Theorem 2.2 is the following. It is well known (for example, by applying the Gärtner-Ellis theorem; see, e.g. [7]) that a large deviation principle holds if the hypotheses of Theorem 2.2 are satisfied, and the rate function is given by the Legendre transform of

$$
\bar{\Lambda}(\lambda)=\frac{1}{2} \int_{0}^{1} \int_{0}^{1} \bar{k}(t, s) \lambda(\mathrm{d} t) \lambda(\mathrm{d} s), \quad \lambda \in \mathcal{M}[0,1] .
$$

In view of Theorem 2.1, we immediately obtain (2.1).

\section{Large deviations for the conditional process}

Let $X=\left(X_{t}\right)_{t \geq 0}$ be a Gaussian, centered process with continuous covariance function

$$
k(t, s)=\operatorname{cov}\left(X_{t}, X_{s}\right)
$$

For a fixed $n \in \mathbb{N}$ and $j=1, \ldots, n$, let $X^{j}=\left(X_{t}^{j}\right)_{t \geq 0}$ stand for the process giving the conditional behavior of $X$ given that it assumes the values $x_{1}, \ldots, x_{j}$ at the $j$ times $0<T_{1}<$ $\cdots<T_{j}$, respectively. Since the original process $X$ is Gaussian, the process $X^{j}=\left(X_{t}^{j}\right)_{t \geq 0}$ is equal in law to (see, e.g. [10])

$$
X_{t}^{j}=X_{t}^{j-1}-\alpha_{j}(t)\left(X_{T_{j}}^{j-1}-x_{j}\right)
$$

where

$$
\alpha_{j}(t)=\frac{k_{j-1}\left(t, T_{j}\right)}{k_{j-1}\left(T_{j}, T_{j}\right)}
$$

and $k_{j}$, which gives the covariance function associated to $X^{j}$, is recursively defined as

$$
\begin{aligned}
k_{j}(t, s) & =\operatorname{cov}\left(X_{t}^{j}, X_{s}^{j}\right) \\
& =k_{j-1}(t, s)-\alpha_{j}(t) k_{j-1}\left(s, T_{j}\right) \\
& =k_{j-1}(t, s)-\alpha_{j}(s) k_{j-1}\left(t, T_{j}\right) .
\end{aligned}
$$

Obviously, the case in which $j=0$ is related to the original process and its covariance function, that is, $X^{0} \equiv X$ and $k_{0} \equiv k$.

Our first aim is to study the behavior of the covariance function of the original process $X$ in order to obtain a functional large deviation principle for the $n$-fold conditional process $X^{n}$ for small time, that is, for $\left\{X_{T_{n}+\varepsilon}^{n}\right\}_{\varepsilon}$ as $\varepsilon \rightarrow 0$. 
Let us consider an infinitesimal function $\gamma_{\varepsilon}\left(\gamma_{\varepsilon} \rightarrow 0\right.$ as $\left.\varepsilon \rightarrow 0\right)$, whose square will play the role of the inverse speed of the large deviation principles we are going to study, and let us introduce the following assumptions.

Assumption 3.1. There exists the asymptotic covariance function $\bar{k}(t, s)$, defined as

$$
\begin{aligned}
\bar{k}(t, s) & =\lim _{\varepsilon \rightarrow 0} \frac{\operatorname{cov}\left(X_{T_{n}+\varepsilon t}-X_{T_{n}}, X_{T_{n}+\varepsilon s}-X_{T_{n}}\right)}{\gamma_{\varepsilon}^{2}} \\
& =\lim _{\varepsilon \rightarrow 0} \frac{k\left(T_{n}+\varepsilon t, T_{n}+\varepsilon s\right)-k\left(T_{n}+\varepsilon t, T_{n}\right)-k\left(T_{n}, T_{n}+\varepsilon s\right)+k\left(T_{n}, T_{n}\right)}{\gamma_{\varepsilon}^{2}}
\end{aligned}
$$

uniformly for $(t, s) \in[0,1] \times[0,1]$.

Assumption 3.2. For any fixed $T>0$, the following limit exists:

$$
\bar{\rho}(t, T)=\lim _{\varepsilon \rightarrow 0} \frac{\operatorname{cov}\left(X_{T_{n}+\varepsilon t}-X_{T_{n}}, X_{T}\right)}{\gamma_{\varepsilon}}=\lim _{\varepsilon \rightarrow 0} \frac{k\left(T_{n}+\varepsilon t, T\right)-k\left(T_{n}, T\right)}{\gamma_{\varepsilon}}
$$

uniformly for $t \in[0,1]$.

Intuitively, Assumption 3.1 defines a 'local process'. In fact, it says that locally, as $\varepsilon \rightarrow 0$, the process $\left(X_{T_{n}+\varepsilon t}-X_{T_{n}}\right)_{t \in[0,1]}$ behaves as a Gaussian process with covariance function given by $\gamma_{\varepsilon}^{2} \bar{k}(t, s)$. Assumption 3.2 is set in order to describe the influence of a distant value on the local process.

Let us discuss some simple but useful consequences of the assumptions introduced above. As an immediate application of Theorem 2.2 (take $U_{t}^{\varepsilon}=X_{T_{n}+\varepsilon t}-X_{T_{n}}$ ), Assumption 3.1 implies that the family $\left\{\left(X_{T_{n}+\varepsilon t}-X_{T_{n}}\right)_{t \in[0,1]}\right\}_{\varepsilon}$ satisfies a large deviation principle on $C([0,1])$ with inverse speed $\gamma_{\varepsilon}^{2}$ and good rate function given by

$$
J(h)= \begin{cases}\frac{1}{2}\|h\|_{\overline{\mathcal{H}}}^{2} & \text { if } h \in \overline{\mathcal{H}}, \\ +\infty & \text { otherwise }\end{cases}
$$

where $\overline{\mathscr{H}}$ is the reproducing kernel Hilbert space associated to the covariance function $\bar{k}(t, s)$ and $\|\cdot\|_{\overline{\mathscr{H}}}$ denotes the usual norm defined on $\overline{\mathscr{H}}$.

Now, in order to achieve a large deviation principle for the $n$-fold conditional process $X^{n}$, we have to investigate the behavior of the functions $k_{j}$, defined through (3.3), in a small time interval of length $\varepsilon$. This can be done by means of Assumption 3.2 as follows.

Lemma 3.1. (i) Under Assumption 3.2, as $j=1, \ldots, n$, we have

$$
\lim _{\varepsilon \rightarrow 0} \frac{\alpha_{j}\left(T_{n}+\varepsilon t\right)-\alpha_{j}\left(T_{n}\right)}{\gamma_{\varepsilon}}=\bar{\alpha}_{j}(t) \quad \text { uniformly for } t \in[0,1] \text {, }
$$

where

$$
\bar{\alpha}_{j}(t)=\frac{\bar{\rho}_{j-1}\left(t, T_{j}\right)}{k_{j-1}\left(T_{j}, T_{j}\right)},
$$

$k_{j-1}$ is as defined in (3.3), $\bar{\rho}_{0} \equiv \bar{\rho}$, and

$$
\begin{aligned}
\bar{\rho}_{j}(t, T) & =\lim _{\varepsilon \rightarrow 0} \frac{k_{j}\left(T_{n}+\varepsilon t, T\right)-k_{j}\left(T_{n}, T\right)}{\gamma_{\varepsilon}} \\
& =\bar{\rho}_{j-1}(t, T)-\bar{\alpha}_{j}(t) k_{j-1}\left(T, T_{j}\right) \\
& =\bar{\rho}_{j-1}(t, T)-\alpha_{j}(T) \bar{\rho}_{j-1}\left(t, T_{j}\right),
\end{aligned}
$$

with the above limit being uniformly for $t \in[0,1]$. 
(ii) Under Assumptions 3.1 and 3.2, we have, for $j=1, \ldots, n$,

$$
\lim _{\varepsilon \rightarrow 0} \mathrm{E}\left(X_{T_{n}+\varepsilon t}^{j}-X_{T_{n}}^{j}\right)=0 \quad \text { uniformly for } t \in[0,1]
$$

and

$$
\lim _{\varepsilon \rightarrow 0} \frac{\operatorname{cov}\left(X_{T_{n}+\varepsilon t}^{j}-X_{T_{n}}^{j}, X_{T_{n}+\varepsilon s}^{j}-X_{T_{n}}^{j}\right)}{\gamma_{\varepsilon}^{2}}=\bar{k}_{j}(t, s) \quad \text { uniformly for } t, s \in[0,1]
$$

with

$$
\bar{k}_{j}(t, s)=\bar{k}(t, s)-\sum_{\ell=1}^{j} k_{\ell-1}\left(T_{\ell}, T_{\ell}\right) \bar{\alpha}_{\ell}(t) \bar{\alpha}_{\ell}(s)
$$

where $\bar{\alpha}_{\ell}$ is as defined in (3.5).

Proof. (i) From Assumption 3.2 and (3.2), we immediately have

$$
\bar{\alpha}_{1}(t)=\frac{\bar{\rho}\left(t, T_{1}\right)}{k\left(T_{1}, T_{1}\right)}
$$

Therefore, by using (3.3), there exists, uniformly for $t \in[0,1]$,

$$
\begin{aligned}
\bar{\rho}_{1}(t, T) & =\lim _{\varepsilon \rightarrow 0} \frac{k_{1}\left(T_{n}+\varepsilon t, T\right)-k_{1}\left(T_{n}, T\right)}{\gamma_{\varepsilon}} \\
& =\bar{\rho}_{0}(t, T)-\bar{\alpha}_{1}(t) k_{0}\left(T, T_{1}\right) \\
& =\bar{\rho}_{0}(t, T)-\alpha_{1}(T) \bar{\rho}_{0}\left(t, T_{1}\right),
\end{aligned}
$$

where, as usual, we have set $\bar{\rho}_{0} \equiv \bar{\rho}$ and $k_{0} \equiv k$. This ensures the existence of $\bar{\alpha}_{2}$. The statement now follows by iteration.

The proof of part (ii) is a straightforward application of Assumption 3.1 and part (i).

Note that, in particular, since $X_{T_{n}}^{n}=x_{n}$, we have, again, uniformly for $t, s \in[0,1]$,

$$
\begin{gathered}
x_{n}=\lim _{\varepsilon \rightarrow 0} \mathrm{E}\left(X_{T_{n}+\varepsilon t}^{n}\right), \\
\bar{k}_{n}(t, s)=\lim _{\varepsilon \rightarrow 0} \frac{\operatorname{cov}\left(X_{T_{n}+\varepsilon t}^{n}, X_{T_{n}+\varepsilon s}^{n}\right)}{\gamma_{\varepsilon}^{2}}=\bar{k}(t, s)-\sum_{\ell=1}^{n} k_{\ell-1}\left(T_{\ell}, T_{\ell}\right) \bar{\alpha}_{\ell}(t) \bar{\alpha}_{\ell}(s) .
\end{gathered}
$$

We are now ready to prove the main large deviation result of this section.

Theorem 3.1. Under Assumptions 3.1 and 3.2, the family $\left\{\left(X_{T_{n}+\varepsilon t}^{n}\right)_{t \in[0,1]}\right\}_{\varepsilon}$ satisfies a large deviation principle on $C([0,1])$ with inverse speed $\gamma_{\varepsilon}^{2}$ and good rate function

$$
J_{n}(h)= \begin{cases}\frac{1}{2}\left\|h-x_{n}\right\|_{\overline{\mathscr{H}}_{n}}^{2} & \text { if } h_{0}=x_{n} \text { and } h-x_{n} \in \overline{\mathscr{H}}_{n}, \\ +\infty & \text { otherwise }\end{cases}
$$


where $\overline{\mathscr{H}}_{n}$ is the reproducing kernel Hilbert space associated to the covariance function

$$
\bar{k}_{n}(t, s)=\bar{k}(t, s)-\sum_{j=1}^{n} k_{j-1}\left(T_{j}, T_{j}\right) \bar{\alpha}_{j}(t) \bar{\alpha}_{j}(s),
$$

where $\bar{k}(\cdot, \cdot), k_{j}(\cdot, \cdot)$, and $\bar{\alpha}_{j}(\cdot)$ are defined in (3.4), (3.3), and (3.5), respectively.

Proof. We start by showing that $\left\{\left(X_{T_{n}+\varepsilon t}^{1}-X_{T_{n}}^{1}\right)_{t \in[0,1]}\right\}_{\varepsilon}$, where $X^{1}$ is as defined in (3.1) with $j=1$, satisfies a large deviation principle. By (3.7), it follows that

$$
\lim _{\varepsilon \rightarrow 0} \mathrm{E}\left(\left\langle\lambda, X_{T_{n}+\varepsilon \cdot}^{1}-X_{T_{n}}^{1}\right\rangle\right)=\lim _{\varepsilon \rightarrow 0} \int_{0}^{1} \mathrm{E}\left(X_{T_{n}+\varepsilon t}^{1}-X_{T_{n}}^{1}\right) \lambda(\mathrm{d} t)=0,
$$

and, recalling that $\operatorname{var}\left(X_{T_{1}}\right)=k\left(T_{1}, T_{1}\right) \equiv k_{0}\left(T_{1}, T_{1}\right)$, by (3.8), it follows that

$$
\begin{aligned}
\lim _{\varepsilon \rightarrow 0} & \frac{\operatorname{var}\left(\left\langle\lambda, X_{T_{n}+\varepsilon \cdot}^{1}-X_{T_{n}}^{1}\right\rangle\right)}{\gamma_{\varepsilon}^{2}} \\
& =\lim _{\varepsilon \rightarrow 0} \int_{0}^{1} \lambda(\mathrm{d} t) \int_{0}^{1} \lambda(\mathrm{d} s) \frac{\operatorname{cov}\left(X_{T_{n}+\varepsilon t}^{1}-X_{T_{n}}^{1}, X_{T_{n}+\varepsilon s}^{1}-X_{T_{n}}^{1}\right)}{\gamma_{\varepsilon}^{2}} \\
& =\int_{0}^{1} \lambda(\mathrm{d} t) \int_{0}^{1} \lambda(\mathrm{d} s)\left(\bar{k}(t, s)-k\left(T_{1}, T_{1}\right) \bar{\alpha}_{1}(t) \bar{\alpha}_{1}(s)\right) .
\end{aligned}
$$

Using Theorem 2.2, we obtain the large deviation principle. Now, iterating the same procedure up to $n$, we would achieve the following (recall that $\operatorname{var}\left(X_{T_{j}}^{j-1}\right)=k_{j-1}\left(T_{j}, T_{j}\right)$ ):

$$
\begin{gathered}
\lim _{\varepsilon \rightarrow 0} \mathrm{E}\left(\left\langle\lambda, X_{T_{n}+\varepsilon}^{n}-X_{T_{n}}^{n}\right\rangle\right)=0, \\
\lim _{\varepsilon \rightarrow 0} \frac{\operatorname{var}\left(\left\langle\lambda, X_{T_{n}+\varepsilon^{*}}^{n}-X_{T_{n}}^{n}\right\rangle\right)}{\gamma_{\varepsilon}^{2}}=\int_{0}^{1} \lambda(\mathrm{d} t) \int_{0}^{1} \lambda(\mathrm{d} s) \bar{k}_{n}(t, s),
\end{gathered}
$$

with $\bar{k}_{n}(t, s)$ as defined in (3.12). Note that, by (3.10), $\bar{k}_{n}$ is a continuous covariance function, being the (uniform) limit of a continuous, symmetric, and positive definite function. Therefore, we can assert that $\left\{\left(X_{T_{n}+\varepsilon t}^{n}-X_{T_{n}}^{n}\right)_{t \in[0,1]}\right\}_{\varepsilon}$ satisfies a large deviation principle on $C([0,1])$ with inverse speed $\gamma_{\varepsilon}^{2}$ and good rate function

$$
H_{n}(\varphi)= \begin{cases}\frac{1}{2}\|\varphi\|_{\overline{\mathscr{H}}_{n}}^{2} & \text { if } \varphi \in \overline{\mathscr{H}}_{n} \\ +\infty & \text { otherwise }\end{cases}
$$

Finally, since $X_{T_{n}+\varepsilon t}^{n}=x_{n}+\left(X_{T_{n}+\varepsilon t}^{n}-X_{T_{n}}^{n}\right)$, the large deviation principle follows by contraction and the associated rate function is actually given by (3.11).

Before we continue with the asymptotic behavior of the $n$-fold conditional bridge process, let us give some examples of applications of Theorem 3.1 to the fractional Brownian motion and integrated Gaussian processes. 


\subsection{Fractional Brownian motion}

The following result holds as a consequence of Theorem 3.1.

Theorem 3.2. Let $X$ be a fractional Brownian motion with Hurst index $H \in(0,1)$, and let $X^{n}$ denote the $n$-fold conditional process as given in (3.1). Then, the family of processes $\left\{\left(X_{T_{n}+\varepsilon t}^{n}\right)_{t \in[0,1]}\right\}_{\varepsilon}$ satisfies a large deviation principle on $C([0,1])$ with inverse speed $\varepsilon^{2 H}$ and good rate function

$$
J_{n}(h)= \begin{cases}\frac{1}{2}\left\|h-x_{n}\right\|_{\mathscr{H}_{H}}^{2} & \text { if } h_{0}=x_{n} \text { and } h-x_{n} \in \mathscr{H}_{H}, \\ +\infty & \text { otherwise, }\end{cases}
$$

where $\mathscr{H}_{H}$ is the reproducing kernel Hilbert space associated to the fractional Brownian motion itself.

Let us recall that a fractional Brownian motion $X$ with Hurst index $H \in(0,1)$ is a continuous, non-Markovian (unless $H=\frac{1}{2}$ ), centered, Gaussian process whose covariance function is

$$
k_{H}(t, s)=\frac{t^{2 H}+s^{2 H}-|t-s|^{2 H}}{2} .
$$

Proof of Theorem 3.2. We show that Assumptions 3.1 and 3.2 hold. First, we have

$$
\frac{\operatorname{cov}\left(X_{T_{n}+\varepsilon t}-X_{T_{n}}, X_{T_{n}+\varepsilon s}-X_{T_{n}}\right)}{\varepsilon^{2 H}}=\operatorname{cov}\left(X_{t}, X_{s}\right),
$$

because of the homogeneity and self-similarity properties holding for the fractional Brownian motion, so that the limit in (3.4) trivially exists and $\bar{k}(t, s)=k_{H}(t, s)$. Concerning Assumption 3.2, straightforward computations (using Taylor's expansion) allow us to easily state that

$$
\lim _{\varepsilon \rightarrow 0} \sup _{t \in[0,1]} \frac{\left|k_{H}\left(T_{n}+\varepsilon t, T\right)-k_{H}\left(T_{n}, T\right)\right|}{\varepsilon^{H}}=0 \quad \text { for any } T>0,
$$

so that $\bar{\rho} \equiv 0$. This in turn implies that $\bar{\alpha}_{j}(t)=0$ for any $t \in[0,1]$ and $j=1, \ldots, n$, as an immediate consequence of what developed in Lemma 3.1(i). Then $\bar{k}_{n} \equiv k$ and the statement now follows from Theorem 3.1.

Note that the $n$-fold conditional fractional Brownian motion satisfies a large deviation principle with the same rate function as the nonconditioned process. This means that the asymptotic behavior of the $n$-fold conditional process does not depend on the past, although $X$ is not Markovian unless $H=\frac{1}{2}$. However, such a local independence property has recently been observed by several authors; see, e.g. [12], as well as [14].

Example 3.1. As an example, let us consider the process

$$
X_{t}=c B_{t}+c_{H} B_{t}^{H},
$$

in which $c$ and $c_{H}$ are non-null real numbers, $B$ stands for a standard Brownian motion, and $B^{H}$ denotes a fractional Brownian motion with Hurst index $H \neq \frac{1}{2}$. Moreover, suppose that $B$ and $B^{H}$ are independent. Such a process has been studied by Cheridito [6], who proved that $X$ is a semimartingale if and only if $H \in\left(\frac{3}{4}, 1\right)$, a property that gives rise to interesting 
applications in finance. The covariance function associated to $X$ is given by

$$
k(t, s)=c^{2} k_{1 / 2}(t, s)+c_{H}^{2} k_{H}(t, s) .
$$

Then, using arguments similar to the ones developed in the proof of Theorem 3.2, we can state a large deviation principle for $\left\{\left(X_{T_{n}+\varepsilon t}^{n}\right)_{t \in[0,1]}\right\}_{\varepsilon}$ on $C([0,1])$ with inverse speed $\varepsilon^{2(H \wedge 1 / 2)}$ and good rate function associated to the covariance function

$$
\bar{k}_{n}(t, s)=\sigma_{H}^{2} k_{H \wedge 1 / 2}(t, s) \quad \text { with } \sigma_{H}^{2}= \begin{cases}c^{2} & \text { if } H>\frac{1}{2}, \\ c_{H}^{2} & \text { if } H<\frac{1}{2},\end{cases}
$$

where $k_{H \wedge 1 / 2}$ denotes the covariance function associated to a fractional Brownian motion with Hurst index $H \wedge \frac{1}{2}$. By contraction, the constant $\sigma_{H}^{2}$ can be put inside the rate function, which becomes

$$
J_{n}(h)= \begin{cases}\frac{1}{2 \sigma_{H}^{2}}\left\|h-x_{n}\right\|_{\mathscr{H}_{H \wedge 1 / 2}}^{2} & \text { if } h_{0}=x_{n} \text { and } h-x_{n} \in \mathscr{H}_{H \wedge 1 / 2}, \\ +\infty & \text { otherwise, }\end{cases}
$$

where $\mathscr{H}_{H \wedge 1 / 2}$ is the reproducing kernel Hilbert space associated with a fractional Brownian motion with Hurst index $H \wedge \frac{1}{2}$.

\subsection{Integrated Gaussian process}

Let $Z$ be a centered Gaussian process with covariance function $\kappa(t, s)$, and let $X$ be the integrated process, i.e.

$$
X_{t}=\int_{0}^{t} Z_{u} \mathrm{~d} u .
$$

The process $X$ is a continuous, centered Gaussian process whose covariance function $k$ is given by

$$
k(t, s)=\int_{0}^{t} \int_{0}^{s} \kappa(u, v) \mathrm{d} u \mathrm{~d} v .
$$

As a consequence of Theorem 3.1, we have the following theorem.

Theorem 3.3. Let $X$ be an integrated Gaussian process, as in $(3.14)$, with $\kappa(t, s)$ continuous, and let $X^{n}$ denote the $n$-fold conditional process given in (3.1). Then, the family $\left\{\left(X_{T_{n}+\varepsilon t}^{n}\right)_{t \in[0,1]}\right\}_{\varepsilon}$ satisfies a large deviation principle on $C([0,1])$ with inverse speed $\varepsilon^{2}$ and good rate function

$$
J_{n}(h)= \begin{cases}\frac{1}{2}\left\|h-x_{n}\right\|_{\overline{\mathcal{H}}_{n}}^{2} & \text { if } h_{0}=x_{n} \text { and } h-x_{n} \in \overline{\mathscr{H}}_{n} \\ +\infty & \text { otherwise }\end{cases}
$$

where $\overline{\mathscr{H}}_{n}$ is the reproducing kernel Hilbert space associated to the covariance function

$$
\bar{k}_{n}(t, s)=a_{n}^{2} t s, \quad \text { where } a_{n}^{2}=\kappa\left(T_{n}, T_{n}\right)-\sum_{j=1}^{n} \frac{d_{j-1}\left(T_{j}\right)^{2}}{k_{j-1}\left(T_{j}, T_{j}\right)},
$$


and $d_{j-1}(T)$ is recursively defined as $d_{0}(T)=\int_{0}^{T} \kappa\left(T_{n}, u\right) \mathrm{d} u$ and

$$
d_{i}(T)=d_{i-1}(T)-\alpha_{i}(T) d_{i-1}\left(T_{i}\right) \text { for } i=1,2, \ldots, n-1
$$

(recall that $k_{j}$ and $\alpha_{j}$ are defined through (3.3) and (3.2), respectively).

Proof. Let us first show that Assumption 3.1 holds with $\gamma_{\varepsilon}=\varepsilon$ and $\bar{k}(t, s)=t s \kappa\left(T_{n}, T_{n}\right)$. In fact,

$$
\begin{aligned}
& \left|\frac{1}{\varepsilon^{2}} \operatorname{cov}\left(X_{T_{n}+\varepsilon t}-X_{T_{n}}, X_{T_{n}+\varepsilon s}-X_{T_{n}}\right)-t s \kappa\left(T_{n}, T_{n}\right)\right| \\
& \quad \leq \frac{1}{\varepsilon^{2}} \int_{T_{n}}^{T_{n}+\varepsilon} \mathrm{d} u \int_{T_{n}}^{T_{n}+\varepsilon} \mathrm{d} v\left|\kappa(u, v)-\kappa\left(T_{n}, T_{n}\right)\right| \\
& \quad \leq \sup _{u, v \in\left[T_{n}, T_{n}+\varepsilon\right]}\left|\kappa(u, v)-\kappa\left(T_{n}, T_{n}\right)\right|,
\end{aligned}
$$

and the last term goes to 0 as $\varepsilon \rightarrow 0$ because $\kappa$ is continuous; thus, $\kappa$ is uniformly continuous on compact sets. Similarly, we prove that Assumption 3.2 also holds with $\bar{\rho}(t, T)=$ $t \int_{0}^{T} \kappa\left(T_{n}, v\right) \mathrm{d} v$. The large deviation principle is now an immediate application of Theorem 3.1. Finally, in order to give the above more explicit expression for $\bar{k}_{n}$, we need the functions $\bar{\alpha}_{j}$. By (3.5), it is sufficient to show that

$$
\bar{\rho}_{j}(t, T)=d_{j}(T) t
$$

We have already seen that $\bar{\rho}_{0}(t, T)=\bar{\rho}(t, T)=d_{0}(T) t$, so that, by (3.6),

$$
\bar{\rho}_{1}(t, T)=\bar{\rho}_{0}(t, T)-\alpha_{1}(T) \bar{\rho}_{0}\left(t, T_{1}\right)=d_{1}(T) t
$$

with $d_{1}(T)=d_{0}(T)-\alpha_{1}(T) d_{0}\left(T_{1}\right)$. By iteration, the statement holds.

Remark 3.1. It follows that the law of an $n$-fold conditional integrated Gaussian process behaves asymptotically as $a_{n} U t$, where $U$ is a standard Gaussian RV. Moreover, a deeper view to the proof of Theorem 3.3 shows that this kind of 'degenerate' behavior can be stated for any Gaussian process whose covariance function $k(t, s)$ is quite smooth, in particular, if both the first and the mixed second derivatives exist, the latter being continuous on the diagonal points $(T, T)$. In fact, in this case the asymptotic covariance $\bar{k}_{n}(t, s)$ for $X^{n}$ is again of the type $a_{n}^{2} t s$.

Example 3.2. ( $m$-fold integrated Brownian motion.) Suppose that $X$ is defined as

$$
X_{t}=\int_{0}^{t} \mathrm{~d} u\left(\int_{0}^{u} \mathrm{~d} u_{m-1} \cdots \int_{0}^{u_{2}} \mathrm{~d} u_{1} W_{u_{1}}\right)
$$

where $W$ denotes a standard Brownian motion. It is known that $X$ is a centered, Gaussian process with covariance function

$$
k(t, s)=\frac{1}{(m !)^{2}} \int_{0}^{s \wedge t}(s-\xi)^{m}(t-\xi)^{m} \mathrm{~d} \xi=\int_{0}^{t} \int_{0}^{s} \kappa(u, v) \mathrm{d} u \mathrm{~d} v
$$

where

$$
\kappa(t, s)=\frac{1}{((m-1) !)^{2}} \int_{0}^{t \wedge s}(t-\xi)^{m-1}(s-\xi)^{m-1} \mathrm{~d} \xi
$$


(for details, see [5]). Then, Theorem 3.3 applies to $X$. Note that, for $T \leq T_{n}$ and $m \geq 1$,

$$
d_{0}(T)=\frac{m}{(m !)^{2}} \int_{0}^{T}\left(T_{n}-\xi\right)^{m-1}(T-\xi)^{m} \mathrm{~d} \xi .
$$

Example 3.3. (Integrated fractional Brownian motion.) Suppose that $X_{t}=\int_{0}^{t} Z_{u} \mathrm{~d} u$, where $Z$ denotes a fractional Brownian motion with Hurst index $H$. Then, the associated covariance function is

$$
k(t, s)=\int_{0}^{t} \int_{0}^{s} \kappa_{H}(u, v) \mathrm{d} u \mathrm{~d} v \quad \text { with } \kappa_{H}(t, s)=\frac{1}{2}\left(t^{2 H}+s^{2 H}-|t-s|^{2 H}\right) .
$$

Again, Theorem 3.3 immediately applies to $X$. Here, for $T \leq T_{n}$, we have

$$
d_{0}(T)=\frac{1}{2}\left(T_{n}^{2 H} T+\frac{1}{2 H+1}\left(T^{2 H+1}-T_{n}^{2 H+1}-\left(T_{n}-T\right)^{2 H+1}\right)\right) .
$$

\section{Large deviations for the bridge of the conditional process}

Let $\left(X_{t}^{n}\right)_{t \geq 0}$ be the $n$-fold conditional process defined in Section 3, and let us now consider the process $Y^{n}$ defined as the bridge of the process $X^{n}$, i.e. the process $X^{n}$ conditioned to be in $y$ at the future time $T_{n}+\varepsilon$. Then, in law, we have

$$
Y_{T_{n}+\varepsilon t}^{n}=X_{T_{n}+\varepsilon t}^{n}-\beta_{T_{n}+\varepsilon t}^{\varepsilon}\left(X_{T_{n}+\varepsilon}^{n}-y\right)
$$

where

$$
\beta_{T_{n}+\varepsilon t}^{\varepsilon}=\frac{k_{n}\left(T_{n}+\varepsilon t, T_{n}+\varepsilon\right)}{k_{n}\left(T_{n}+\varepsilon, T_{n}+\varepsilon\right)} .
$$

Now, in order to achieve a large deviation principle for $\left\{\left(Y_{T_{n}+\varepsilon t}^{n}\right)_{t \in[0,1]}\right\}_{\varepsilon}$, we need a suitable asymptotic behavior for $\beta_{T_{n}+\varepsilon}^{\varepsilon}$. In fact, we have the following lemma.

Lemma 4.1. Let Assumptions 3.1 and 3.2 be satisfied. Then there exists the limit

$$
\lim _{\varepsilon \rightarrow 0} \beta_{T_{n}+\varepsilon t}^{\varepsilon}=\frac{\bar{k}_{n}(t, 1)}{\bar{k}_{n}(1,1)}=: \bar{\beta}_{t} \quad \text { uniformly for } t \in[0,1] .
$$

Proof. We have

$$
\begin{aligned}
\left|\beta_{T_{n}+\varepsilon t}^{\varepsilon}-\bar{\beta}_{t}\right|= & \left|\frac{k_{n}\left(T_{n}+\varepsilon t, T_{n}+\varepsilon\right)}{k_{n}\left(T_{n}+\varepsilon, T_{n}+\varepsilon\right)}-\frac{\bar{k}_{n}(t, 1)}{\bar{k}_{n}(1,1)}\right| \\
\leq & \frac{\gamma_{\varepsilon}^{2}}{k_{n}\left(T_{n}+\varepsilon, T_{n}+\varepsilon\right)}\left|\frac{k_{n}\left(T_{n}+\varepsilon t, T_{n}+\varepsilon\right)}{\gamma_{\varepsilon}^{2}}-\bar{k}_{n}(t, 1)\right| \\
& +|\bar{k}(t, 1)|\left|\frac{\gamma_{\varepsilon}^{2}}{k_{n}\left(T_{n}+\varepsilon, T_{n}+\varepsilon\right)}-\frac{1}{\bar{k}_{n}(1,1)}\right| .
\end{aligned}
$$

From (3.10),

$$
\begin{aligned}
& \lim _{\varepsilon \rightarrow 0} \sup _{t \in[0,1]}\left|\frac{k_{n}\left(T_{n}+\varepsilon t, T_{n}+\varepsilon\right)}{\gamma_{\varepsilon}^{2}}-\bar{k}_{n}(t, 1)\right|=0 \\
& \text { and } \lim _{\varepsilon \rightarrow 0}\left|\frac{\gamma_{\varepsilon}^{2}}{k_{n}\left(T_{n}+\varepsilon, T_{n}+\varepsilon\right)}-\frac{1}{\bar{k}_{n}(1,1)}\right|=0,
\end{aligned}
$$

so that the statement holds. 
It is now easy to prove a first large deviation principle. But, as we will see, there are cases in which the next, immediate, result turns out to be degenerate in some sense. So, let us split this section into two subsections: Subsection 4.1 contains a first result and Subsection 4.2 develops some refinements.

\subsection{A first large deviation result for the bridge}

Theorem 4.1. Let $Y^{n}$ be the bridge of the $n$-fold conditional process $X^{n}$, as defined in (4.1). Under Assumptions 3.1 and 3.2, the family of processes $\left\{\left(Y_{T_{n}+\varepsilon t}^{n}\right)_{t \in[0,1]}\right\}_{\varepsilon}$ satisfies a large deviation principle on $C([0,1])$ with inverse speed $\gamma_{\varepsilon}^{2}$ and good rate function

$$
J_{Y}(h)= \begin{cases}\frac{1}{2}\|h-\bar{m}\|_{\overline{\mathcal{H}}_{Y}}^{2} & \text { if } h_{0}=x_{n}, h_{1}=y, h-\bar{m} \in \overline{\mathscr{H}}_{Y}, \\ +\infty & \text { otherwise, }\end{cases}
$$

where $\bar{m}_{t}=x_{n}+\bar{\beta}_{t}\left(y-x_{n}\right)$ and $\overline{\mathscr{H}}_{Y}$ is the reproducing kernel Hilbert space associated to the covariance function

$$
\bar{k}_{Y}(t, s)=\bar{k}_{n}(t, s)-\bar{\beta}_{s} \bar{k}_{n}(t, 1)=\bar{k}_{n}(t, s)-\frac{\bar{k}_{n}(t, 1) \bar{k}_{n}(s, 1)}{\bar{k}_{n}(1,1)} .
$$

Proof. First, let us set

$$
U_{T_{n}+\varepsilon t}^{n}=Y_{T_{n}+\varepsilon t}^{n}-\bar{m}_{t}, \quad \text { where } \bar{m}_{t}=x_{n}+\bar{\beta}_{t}\left(y-x_{n}\right)=\lim _{\varepsilon \rightarrow 0} \mathrm{E}\left(Y_{T_{n}+\varepsilon t}^{n}\right),
$$

and note that, by (3.9), (4.1), and (4.2), the above limit holds uniformly for $t \in[0,1]$. We will start by showing a large deviation principle for $\left\{U_{T_{n}+\varepsilon}^{n} \text {. }\right\}_{\varepsilon}$, again by using Theorem 2.2. In fact,

$$
\lim _{\varepsilon \rightarrow 0} \mathrm{E}\left(\left\langle\lambda, U_{T_{n}+\varepsilon}^{n} .\right\rangle\right)=\int_{0}^{1} \lambda(\mathrm{d} t) \mathrm{E}\left(U_{T_{n}+\varepsilon t}^{n}\right)=0 \quad \text { for any } \lambda \in \mathcal{M}[0,1] .
$$

Moreover, from (3.10) and Lemma 4.1, we have

$$
\begin{aligned}
\lim _{\varepsilon \rightarrow 0} \frac{\operatorname{cov}\left(U_{T_{n}+\varepsilon t}^{n}, U_{T_{n}+\varepsilon s}^{n}\right)}{\gamma_{\varepsilon}^{2}} & =\lim _{\varepsilon \rightarrow 0} \frac{\operatorname{cov}\left(Y_{T_{n}+\varepsilon t}^{n}, Y_{T_{n}+\varepsilon s}^{n}\right)}{\gamma_{\varepsilon}^{2}} \\
& =\lim _{\varepsilon \rightarrow 0} \frac{\operatorname{cov}\left(X_{T_{n}+\varepsilon t}^{n}, X_{T_{n}+\varepsilon s}^{n}\right)-\beta_{T_{n}+\varepsilon s}^{\varepsilon} \operatorname{cov}\left(X_{T_{n}+\varepsilon t}^{n}, X_{T_{n}+\varepsilon}^{n}\right)}{\gamma_{\varepsilon}^{2}} \\
& =\bar{k}_{n}(t, s)-\bar{\beta}_{s} \bar{k}_{n}(t, 1) \\
& =: \bar{k}_{Y}(t, s) \quad \text { uniformly for } s, t \in[0,1],
\end{aligned}
$$

so that

$$
\begin{aligned}
\lim _{\varepsilon \rightarrow 0} \frac{\operatorname{var}\left(\left\langle\lambda, U_{T_{n}+\varepsilon .}^{n}\right\rangle\right)}{\gamma_{\varepsilon}^{2}} & =\lim _{\varepsilon \rightarrow 0} \frac{1}{\gamma_{\varepsilon}^{2}} \int_{0}^{1} \lambda(\mathrm{d} t) \int_{0}^{1} \lambda(\mathrm{d} s) \operatorname{cov}\left(U_{T_{n}+\varepsilon t}^{n} U_{T_{n}+\varepsilon s}^{n}\right) \\
& =\int_{0}^{1} \lambda(\mathrm{d} t) \int_{0}^{1} \lambda(\mathrm{d} s) \bar{k}_{Y}(t, s) \quad \text { for any } \lambda \in \mathcal{M}[0,1] .
\end{aligned}
$$

We can then assert that the family of processes $\left\{\left(U_{T_{n}+\varepsilon t}^{n}\right)_{t \in[0,1]}\right\}_{\varepsilon}$ does satisfy a large deviation principle on $C([0,1])$ with inverse speed $\gamma_{\varepsilon}^{2}$ and good rate function

$$
J_{U}(\varphi)= \begin{cases}\frac{1}{2}\|\varphi\|_{\overline{\mathscr{H}}_{Y}}^{2} & \text { if } \varphi_{0}=\varphi_{1}=0 \text { and } \varphi \in \overline{\mathscr{H}}_{Y}, \\ +\infty & \text { otherwise, }\end{cases}
$$


where $\overline{\mathscr{H}}_{Y}$ is the reproducing kernel Hilbert space associated to the covariance function

$$
\bar{k}_{Y}(t, s)=\bar{k}_{n}(t, s)-\bar{\beta}_{s} \bar{k}_{n}(t, 1)=\bar{k}_{n}(t, s)-\frac{\bar{k}_{n}(t, 1) \bar{k}_{n}(s, 1)}{\bar{k}_{n}(1,1)} .
$$

Let us stress that the condition $\varphi_{0}=\varphi_{1}=0$ is trivially satisfied if $\varphi \in \overline{\mathcal{H}}_{Y}$ (it immediately follows from the fact that $k_{Y}(0, s)=k_{Y}(1, s)=0$ for any $\left.s\right)$, but we have chosen to write it for the sake of clearness. Now, since $Y_{T_{n}+\varepsilon t}^{n}=U_{T_{n}+\varepsilon t}^{n}+\bar{m}_{t}$, by contraction, we immediately obtain the large deviation principle for $\left\{\left(Y_{T_{n}+\varepsilon t}^{n}\right)_{t \in[0,1]}\right\}_{\varepsilon}$ on $C([0,1])$ with inverse speed $\gamma_{\varepsilon}^{2}$ and good rate function as in (4.3).

Remark 4.1. The rate function $J_{Y}$ given by (4.3) can also be written in the following way:

$$
J_{Y}(h)= \begin{cases}\frac{1}{2}\left(\left\|h-x_{n}\right\|_{\overline{\mathcal{H}}_{n}}^{2}-\frac{\left(y-x_{n}\right)^{2}}{\bar{k}_{n}(1,1)}\right) & \text { if } h_{0}=x_{n}, h_{1}=y, \text { and } h-x_{n} \in \overline{\mathscr{H}}_{n}, \\ +\infty & \text { otherwise }\end{cases}
$$

where $\overline{\mathscr{H}}_{n}$ is the reproducing kernel Hilbert space associated to the covariance function $\bar{k}_{n}$ defined in (3.12). Such a representation agrees with well-known formulae, for example, whenever $X$ is a standard Brownian motion (see, e.g. [4]). The proof of (4.4) is postponed to Appendix A.

Example 4.1. (Fractional Brownian motion.) Following Subsection 3.1, let $X$ be a fractional Brownian motion with Hurst index $H$ and let $X^{n}$ be the associated $n$-fold conditional process. As seen in Theorem 3.2, Assumptions 3.1 and 3.2 hold and the asymptotic covariance function $\bar{k}_{n}(t, s)$ coincides with the original covariance function $k_{H}(t, s)$. By applying Theorem 4.1, the bridge process $Y^{n}$ satisfies a functional large deviation principle for small time with inverse speed $\varepsilon^{2 H}$ and good rate function

$$
J_{Y}(h)= \begin{cases}\frac{1}{2}\|h-\bar{m}\|_{\overline{\mathcal{H}}_{Y}}^{2} & \text { if } h_{0}=x_{n}, h_{1}=y, \text { and } h-x_{n} \in \overline{\mathcal{H}}_{Y}, \\ +\infty & \text { otherwise }\end{cases}
$$

where $\overline{\mathscr{H}}_{Y}$ is the reproducing kernel Hilbert space associated to the covariance function

$$
\bar{k}_{Y}(t, s)=k_{H}(t, s)-k_{H}(t, 1) k_{H}(1, s) .
$$

Using (4.4), $J_{Y}$ can also be written in terms of the reproducing kernel Hilbert space $\mathscr{H}_{H}$ associated to the original fractional Brownian motion $X$ :

$$
J_{Y}(h)= \begin{cases}\frac{1}{2}\left(\left\|h-x_{n}\right\|_{\mathscr{H}_{H}}^{2}-\left(y-x_{n}\right)^{2}\right) & \text { if } h_{0}=x_{n}, h_{1}=y, \text { and } h-x_{n} \in \mathscr{H}_{H}, \\ +\infty & \text { otherwise }\end{cases}
$$

Whenever $H=\frac{1}{2}$, that is, $X$ is a standard Brownian motion, then the above result is well known and widely applied in the literature. Moreover, (4.5) confirms that, as a consequence of the 'local' independence of the $n$-fold conditional fractional Brownian motion, its bridge also satisfies a large deviation principle which is independent of all the past except for what happens at time $T_{n}$.

Example 4.2. (Cheridito process.) Let $X$ be the process given in Example 3.1, i.e. $X_{t}=$ $c B_{t}+c_{H} B_{t}^{H}$, where $c, c_{H} \neq 0$ are constant numbers, $B$ and $B^{H}$ are independent, $B$ denotes 
a Brownian motion, and $B^{H}$ denotes a fractional Brownian motion with Hurst index $H \neq \frac{1}{2}$. By developing arguments similar to the ones in Example 4.1, we find that the bridge of the associated $n$-fold conditional process satisfies a large deviation principle. By taking into account the results in Example 3.1 and (4.4), we easily find that the inverse speed is equal to $\varepsilon^{2(H \wedge 1 / 2)}$ and the good rate function is given by the following formula:

$J_{Y}(h)= \begin{cases}\frac{1}{2 \sigma_{H}^{2}}\left(\left\|h-x_{n}\right\|_{\mathcal{H}_{H \wedge 1 / 2}^{2}}-\left(y-x_{n}\right)^{2}\right) & \text { if } h_{0}=x_{n}, h_{1}=y, \text { and } h-x_{n} \in \mathscr{H}_{H \wedge 1 / 2}, \\ +\infty & \text { otherwise, }\end{cases}$

where $\sigma_{H}^{2}$ is given by (3.13).

Example 4.3. (Integrated Gaussian processes.) Following Subsection 3.2, let $X^{n}$ be the $n$-fold conditional process when $X$ is an integrated Gaussian process, as in (3.14). Under the hypotheses of Theorem 3.3, Assumptions 3.1 and 3.2 hold, and a functional large deviation principle for $X^{n}$ follows, with asymptotic covariance function $\bar{k}_{n}(t, s)=a_{n}^{2} t s$ for a suitable constant $a_{n}^{2}$. Now, by applying Theorem 4.1, we obtain a functional large deviation principle for the bridge process $Y^{n}$ as well, but unfortunately we obtain a degenerate asymptotic behavior because the associated rate function turns out to be

$$
J_{Y}(h)= \begin{cases}0 & \text { if } h=\bar{m} \\ +\infty & \text { otherwise. }\end{cases}
$$

This follows from the fact that, since $\overline{\mathscr{H}}_{n}$ is 'spanned' by the covariance function $\bar{k}_{n}(t, s)=a_{n}^{2} t s$, it contains only the paths running at constant speed. Then, $J_{Y}$ is finite only for $h$ such that $h-\bar{m}=c t$. Since here $\bar{m}_{t}=x_{n}+\left(y-x_{n}\right) t$, the additional constraints $h_{0}=x_{n}$ and $h_{1}=y$ give the unique path $h=\bar{m}$.

Note that Theorem 4.1 gives an unsatisfactory large deviation result not only for integrated Gaussian processes but also for Gaussian processes whose (original) covariance function is smooth enough; as observed in Remark 3.1, in this case the asymptotic covariance function is (constant)ts as well, and the same degenerate behavior holds for the rate function. This motivates the next subsection, in which we study some refinements that allow us to state nontrivial large deviation estimates or, more precisely, the correct large deviation speed.

\subsection{Faster large deviations for the bridge}

In this subsection we prove a refined version of Theorem 4.1: we study the exact (faster) speed giving a nontrivial rate function whenever the covariance is smooth. With the same notation as Section 3, Assumptions 3.1 and 3.2 must be strengthened here as follows.

Assumption 4.1. For some $\alpha \in(0,1]$,

(i) there exist a function $\bar{\varphi}(t, s)$, a constant $a^{2}$, and a remaining term $\mathcal{R}_{\varepsilon}^{1}(t, s)$ (depending on $T_{n}$ ) such that

$$
\operatorname{cov}\left(X_{T_{n}+\varepsilon t}-X_{T_{n}}, X_{T_{n}+\varepsilon s}-X_{T_{n}}\right)=\varepsilon^{2}\left(a^{2} t s+\bar{\varphi}(t, s) \varepsilon^{\alpha}+\mathcal{R}_{\varepsilon}^{1}(t, s)\right)
$$

with

$$
\lim _{\varepsilon \rightarrow 0} \sup _{s, t \in[0,1]} \frac{\left|\mathcal{R}_{\varepsilon}^{1}(t, s)\right|}{\varepsilon^{\alpha}}=0
$$


(ii) for any fixed $T>0$, there exist a function $\bar{\psi}(t, T)$, a constant $c(T)$, and a remaining term $\mathcal{R}_{\varepsilon}^{2}(t ; T)$ (depending on $T_{n}$ ) such that

$$
k\left(T_{n}+\varepsilon t, T\right)-k\left(T_{n}, T\right)=\varepsilon\left(c(T) t+\bar{\psi}(t ; T) \varepsilon^{\alpha}+\mathcal{R}_{\varepsilon}^{2}(t ; T)\right)
$$

with

$$
\lim _{\varepsilon \rightarrow 0} \sup _{t \in[0,1]} \frac{\left|\mathcal{R}_{\varepsilon}^{2}(t ; T)\right|}{\varepsilon^{\alpha}}=0 .
$$

As a consequence of Assumption 4.1, by using the same arguments as in Lemma 3.1 we immediately prove the following lemma.

Lemma 4.2. For $j=1, \ldots, n$,

$$
k_{j}\left(T_{n}+\varepsilon t\right)-k_{j}\left(T_{n}, T\right)=\varepsilon\left(c_{j}(T) t+\bar{\psi}_{j}(t ; T) \varepsilon^{\alpha}+\mathcal{R}_{\varepsilon}^{2}(t ; T)\right),
$$

where $c_{0} \equiv c, \bar{\psi}_{0} \equiv \bar{\psi}$, and $c_{j}$ and $\bar{\psi}_{j}$ are given by

$$
c_{j}(T)=c_{j-1}(T)-\alpha_{j}(T) c_{j-1}\left(T_{j}\right) \quad \text { and } \quad \bar{\psi}_{j}(t ; T)=\bar{\psi}_{j-1}(t ; T)-\alpha_{j}(T) \bar{\psi}_{j-1}\left(t ; T_{j}\right) .
$$

Moreover,

$$
\operatorname{cov}\left(X_{T_{n}+\varepsilon t}^{j}-X_{T_{n}}^{j}, X_{T_{n}+\varepsilon s}^{j}-X_{T_{n}}^{j}\right)=\varepsilon^{2}\left(a_{j}^{2} t s+\bar{\varphi}_{j}(t, s) \varepsilon^{\alpha}+\mathcal{R}_{\varepsilon}^{1, j}(t, s)\right),
$$

where $\mathcal{R}_{\varepsilon}^{1, j}(t, s) \rightarrow 0$ as $\varepsilon \rightarrow 0$ uniformly on $[0,1] \times[0,1], a_{j}=a-\sum_{\ell=1}^{j} c_{\ell-1}^{2}\left(T_{\ell}\right)$, and

$$
\bar{\varphi}_{j}(t, s)=\bar{\varphi}(t, s)-\sum_{\ell=1}^{j} \frac{c_{\ell-1}\left(T_{\ell}\right)}{k_{\ell-1}\left(T_{\ell}, T_{\ell}\right)}\left(\bar{\psi}_{\ell-1}\left(t ; T_{\ell}\right) s+\bar{\psi}_{\ell-1}\left(s ; T_{\ell}\right) t\right) .
$$

In particular, since $X_{T_{n}}^{n}=x_{n}$,

$k_{n}\left(T_{n}+\varepsilon t, T_{n}+\varepsilon s\right)=\varepsilon^{2}\left(a_{n}^{2} t s+\bar{\varphi}_{n}(t, s) \varepsilon^{\alpha}+\mathcal{R}_{\varepsilon}^{1, n}(t, s)\right) \quad$ with $\lim _{\varepsilon \rightarrow 0} \sup _{t, s \in[0,1]} \frac{\left|\mathcal{R}_{\varepsilon}^{1, n}(t, s)\right|}{\varepsilon^{\alpha}}=0$.

Then, we have the following theorem.

Theorem 4.2. Let $Y^{n}$ be the bridge of the $n$-fold conditional process $X^{n}$, as defined in (4.1). If Assumption 4.1 holds then the family of processes $\left\{\left(Y_{T_{n}+\varepsilon t}^{n}\right)_{t \in[0,1]}\right\}_{\varepsilon}$ satisfies a large deviation principle on $C([0,1])$ with inverse speed $\varepsilon^{2+\alpha}$ and good rate function

$$
J_{Y}(h)= \begin{cases}\frac{1}{2}\|h-\bar{m}\|_{\overline{\mathscr{H}}_{Y}}^{2} & \text { if } h-\bar{m} \in \overline{\mathscr{H}}_{Y} \\ +\infty & \text { otherwise }\end{cases}
$$

where $\bar{m}_{t}=x_{n}+\bar{\beta}_{t}\left(y-x_{n}\right)$ and $\overline{\mathscr{H}}_{Y}$ is the reproducing kernel Hilbert space associated to the covariance function

$$
\bar{k}_{Y}(t, s)=\bar{\varphi}_{n}(t, s)+t s \bar{\varphi}_{n}(1,1)-t \bar{\varphi}_{n}(1, s)-s \bar{\varphi}_{n}(t, 1) .
$$


Proof. The proof is the same as Theorem 4.1. It is enough to observe that in this case we have, from (4.7),

$$
\begin{aligned}
\operatorname{cov}( & \left.Y_{T_{n}+\varepsilon t}^{n}, Y_{T_{n}+\varepsilon s}^{n}\right) \\
= & k_{n}\left(T_{n}+\varepsilon t, T_{n}+\varepsilon s\right)-\frac{k_{n}\left(T_{n}+\varepsilon t, T_{n}+\varepsilon\right) k_{n}\left(T_{n}+\varepsilon, T_{n}+\varepsilon s\right)}{k_{n}\left(T_{n}+\varepsilon, T_{n}+\varepsilon\right)} \\
= & \varepsilon^{2}\left(a_{n}^{2} t s+\bar{\varphi}_{n}(t, s) \varepsilon^{\alpha}-\frac{\left(a_{n}^{2} t+\bar{\varphi}_{n}(t, 1) \varepsilon^{\alpha}\right)\left(a_{n}^{2} s+\bar{\varphi}_{n}(1, s) \varepsilon^{\alpha}\right)}{a_{n}^{2}+\bar{\varphi}_{n}(1,1) \varepsilon^{\alpha}+\mathcal{R}_{\varepsilon}^{1, n}(1,1)}+\mathcal{R}_{\varepsilon}^{1, n}(t, s)\right) \\
= & \frac{a_{n}^{2} \varepsilon^{2}}{a_{n}^{2}+\bar{\varphi}_{n}(1,1) \varepsilon^{\alpha}+\mathcal{R}_{\varepsilon}^{1, n}(1,1)} \\
& \times\left(\left(\bar{\varphi}_{n}(t, s)+t s \bar{\varphi}_{n}(1,1)-t \bar{\varphi}_{n}(1, s)-s \bar{\varphi}_{n}(t, 1)\right) \varepsilon^{\alpha}+\mathcal{R}_{\varepsilon}^{1, n}(t, s)\right) .
\end{aligned}
$$

Therefore,

$$
\lim _{\varepsilon \rightarrow 0} \frac{\operatorname{cov}\left(Y_{T_{n}+\varepsilon t}^{n}, Y_{T_{n}+\varepsilon s}^{n}\right)}{\varepsilon^{2+\alpha}}=\bar{\varphi}_{n}(t, s)+t s \bar{\varphi}_{n}(1,1)-t \bar{\varphi}_{n}(1, s)-s \bar{\varphi}_{n}(t, 1),
$$

uniformly for $s, t \in[0,1]$, and the theorem holds.

Remark 4.2. Note that $\bar{\varphi}_{n}$ is symmetric and continuous whereas it is not positive definite in general, so that it is not necessarily a covariance function. Nevertheless, $k_{Y}$ given by (4.8) does represent a covariance function, as an immediate consequence of (4.9). However, if $\bar{\varphi}_{n}$ was a covariance function, a curious effect would happen: the asymptotic behavior of the bridge is regulated by a covariance function which coincides with the one associated to what is usually called 'the false bridge', that is, a process of the type $Z_{t}-t Z_{1}$, where $Z$ is a Gaussian process with covariance $\bar{\varphi}_{n}$.

Now, if the function $k(t, s)$ is more regular then Theorem 4.2 would again give a degenerate behavior. In fact, suppose that $k$ has continuous derivatives up to the third order. Then, since $k$ is symmetric, by straightforward computations we obtain

$$
\bar{\varphi}(t, s)=\frac{1}{3 !}\left(3 \partial_{t t s}^{3} k\left(T_{n}, T_{n}\right) t^{2} s+3 \partial_{t s s}^{3} k\left(T_{n}, T_{n}\right) t s^{2}\right)=\frac{1}{2} \partial_{t t s}^{3} k\left(T_{n}, T_{n}\right) t s(t+s),
$$

and by using (4.6) we can show that $\bar{\varphi}_{n}(t, s)=b_{n} t s(t+s)$ for a suitable constant $b_{n}$. Therefore, by (4.8) we have

$$
\bar{k}_{Y}(t, s)=b_{n} t s(t+s)+2 b_{n} t s-b_{n}\left(t^{2}+t\right) s-b_{n}\left(s+s^{2}\right) t \equiv 0,
$$

and again a trivial large deviation principle holds for the bridge of the conditional process. Let us refine further on the hypothesis.

Assumption 4.2. For some $\alpha \in(0,1]$,

(i) there exist a function $\bar{\varphi}(t, s)$, constants $a^{2}$ and $b$, and a remaining term $\mathcal{R}_{\varepsilon}^{1}(t, s)$ (depending on $T_{n}$ ) such that

$$
\begin{aligned}
& \operatorname{cov}\left(X_{T_{n}+\varepsilon t}-X_{T_{n}}, X_{T_{n}+\varepsilon s}-X_{T_{n}}\right) \\
& \quad=\varepsilon^{2}\left(a^{2} t s+b\left(t^{2} s+t s^{2}\right) \varepsilon+\bar{\varphi}(t, s) \varepsilon^{1+\alpha}+\mathcal{R}_{\varepsilon}^{1}(t, s)\right)
\end{aligned}
$$


with

$$
\lim _{\varepsilon \rightarrow 0} \sup _{s, t \in[0,1]} \frac{\left|\mathcal{R}_{\varepsilon}^{1}(t, s)\right|}{\varepsilon^{1+\alpha}}=0 ;
$$

(ii) for any fixed $T>0$, there exist a function $\bar{\psi}(t, T)$, constants $c(T)$ and $d(T)$, and a remaining term $\mathcal{R}_{\varepsilon}^{2}(t ; T)$ (depending on $T_{n}$ ) such that

$$
k\left(T_{n}+\varepsilon t, T\right)-k\left(T_{n}, T\right)=\varepsilon\left(c(T) t+d(T) t^{2} \varepsilon+\bar{\psi}(t ; T) \varepsilon^{1+\alpha}+\mathcal{R}_{\varepsilon}^{2}(t ; T)\right)
$$

with

$$
\lim _{\varepsilon \rightarrow 0} \sup _{t \in[0,1]} \frac{\left|\mathcal{R}_{\varepsilon}^{2}(t ; T)\right|}{\varepsilon^{1+\alpha}}=0 .
$$

Let us remark that if $k(t, s)$ is smooth enough then we immediately have $a^{2}=\partial_{t s}^{2} k\left(T_{n}, T_{n}\right)$, $b=\frac{1}{2} \partial_{t t s}^{3} k\left(T_{n}, T_{n}\right), c(T)=\partial_{t} k\left(T_{n}, T\right)$, and $d(T)=\frac{1}{2} \partial_{t t}^{2} k\left(T_{n}, T\right)$.

Moreover, as an immediate consequence of Assumption 4.2, by using the same arguments as in Lemma 3.1 and Lemma 4.2, we are able to prove the following lemma.

Lemma 4.3. For $j=1, \ldots, n$,

$$
k_{j}\left(T_{n}+\varepsilon t\right)-k_{j}\left(T_{n}, T\right)=\varepsilon\left(c_{j}(T) t+d_{j}(T) t^{2} \varepsilon+\bar{\psi}_{j}(t ; T) \varepsilon^{1+\alpha}+\mathcal{R}_{\varepsilon}^{2, j}(t ; T)\right),
$$

where $c_{0}(T)=c(T), d_{0}(T)=d(T), \bar{\psi}_{0} \equiv \bar{\psi}$, and $c_{j}(T), d_{j}(T)$, and $\bar{\psi}_{j}$ are defined as follows:

$$
\begin{aligned}
c_{j}(T) & =c_{j-1}(T)-\alpha_{j}(T) c_{j-1}\left(T_{j}\right), \\
d_{j}(T) & =d_{j-1}(T)-\alpha_{j}(T) d_{j-1}\left(T_{j}\right), \\
\bar{\psi}_{j}(t ; T) & =\bar{\psi}_{j-1}(t ; T)-\alpha_{j}(T) \bar{\psi}_{j-1}\left(t ; T_{j}\right) .
\end{aligned}
$$

Moreover,

$\operatorname{cov}\left(X_{T_{n}+\varepsilon t}^{j}-X_{T_{n}}^{j}, X_{T_{n}+\varepsilon s}^{j}-X_{T_{n}}^{j}\right)=\varepsilon^{2}\left(a_{j}^{2} t s+b_{j}\left(t^{2} s+t s^{2}\right) \varepsilon+\bar{\varphi}_{j}(t, s) \varepsilon^{1+\alpha}+\mathcal{R}_{\varepsilon}^{1, j}(t, s)\right)$, where $a_{j}^{2}=a^{2}-\sum_{\ell=1}^{j} c_{\ell-1}^{2}\left(T_{\ell}\right), b_{j}=b-\sum_{\ell=1}^{j} c_{\ell-1}\left(T_{\ell}\right) d_{\ell-1}\left(T_{\ell}\right)$, and

$$
\bar{\varphi}_{j}(t, s)= \begin{cases}\bar{\varphi}(t, s)-\sum_{\ell=1}^{j} \frac{c_{\ell-1}\left(T_{\ell}\right)}{k_{\ell-1}\left(T_{\ell}, T_{\ell}\right)}\left(\bar{\psi}_{\ell-1}\left(t ; T_{\ell}\right) s+\bar{\psi}_{\ell-1}\left(s ; T_{\ell}\right) t\right) & \text { for } \alpha<1, \\ \bar{\varphi}(t, s)-\sum_{\ell=1}^{j} \frac{c_{\ell-1}\left(T_{\ell}\right)}{k_{\ell-1}\left(T_{\ell}, T_{\ell}\right)}\left(\bar{\psi}_{\ell-1}\left(t ; T_{\ell}\right) s+\bar{\psi}_{\ell-1}\left(s ; T_{\ell}\right) t\right) & \text { for } \alpha=1 . \\ -\sum_{\ell=1}^{j} \frac{d_{\ell-1}^{2}\left(T_{\ell}\right)}{k_{\ell-1}\left(T_{\ell}, T_{\ell}\right)} t^{2} s^{2} & \end{cases}
$$

In particular, since $X_{T_{n}}^{n}=x_{n}$, we have

$$
k_{n}\left(T_{n}+\varepsilon t, T_{n}+\varepsilon s\right)=\varepsilon^{2}\left(a_{n} t s+b_{n}\left(t^{2} s+t s^{2}\right) \varepsilon+\bar{\varphi}_{n}(t, s) \varepsilon^{1+\alpha}+\mathcal{R}_{\varepsilon}^{1, n}(t, s)\right) .
$$

Let us stress that in Lemma 4.3, the notation $\mathcal{R}_{\varepsilon}$ (with some suitable superscript) stands for a generic remaining term, which uniformly converges to 0 as $\varepsilon \rightarrow 0$.

Then, we have the following theorem. 
Theorem 4.3. Let $Y^{n}$ be the bridge of the $n$-fold conditional process $X^{n}$, as defined in (4.1). If Assumption 4.2 holds then the family of processes $\left\{\left(Y_{T_{n}+\varepsilon t}^{n}\right)_{t \in[0,1]}\right\}_{\varepsilon}$ satisfies a large deviation principle on $C([0,1])$ with inverse speed $\varepsilon^{3+\alpha}$ and good rate function

$$
J_{Y}(h)= \begin{cases}\frac{1}{2}\|h-\bar{m}\|_{\overline{\mathcal{H}}_{Y}}^{2} & \text { if } h-\bar{m} \in \overline{\mathcal{H}}_{Y}, \\ +\infty & \text { otherwise, }\end{cases}
$$

where $\bar{m}_{t}=x_{n}+\bar{\beta}_{t}\left(y-x_{n}\right)$ and $\overline{\mathscr{H}}_{Y}$ is the reproducing kernel Hilbert space associated to the covariance function

$$
\bar{k}_{Y}(t, s)= \begin{cases}\bar{\varphi}_{n}(t, s)+t s \bar{\varphi}_{n}(1,1)-t \bar{\varphi}_{n}(1, s)-s \bar{\varphi}_{n}(t, 1) & \text { if } \alpha<1, \\ b_{n}^{2}\left(t s^{2}+t^{2} s-t^{2} s^{2}-s t\right)+\bar{\varphi}_{n}(t, s)+t s \bar{\varphi}_{n}(1,1) & \\ -t \bar{\varphi}_{n}(1, s)-s \bar{\varphi}_{n}(t, 1) & \text { if } \alpha=1 .\end{cases}
$$

Proof. The proof is the same as Theorem 4.1. It is enough to observe that

$$
\begin{aligned}
\operatorname{cov}\left(Y_{T_{n}+\varepsilon t}^{n}, Y_{T_{n}+\varepsilon s}^{n}\right) & \\
= & k_{n}\left(T_{n}+\varepsilon t, T_{n}+\varepsilon s\right)-\frac{k_{n}\left(T_{n}+\varepsilon t, T_{n}+\varepsilon\right) k_{n}\left(T_{n}+\varepsilon, T_{n}+\varepsilon s\right)}{k_{n}\left(T_{n}+\varepsilon, T_{n}+\varepsilon\right)} \\
= & \varepsilon^{2}\left(a_{n}^{2} t s+b_{n}\left(t^{2} s+t s^{2}\right) \varepsilon+\bar{\varphi}_{n}(t, s) \varepsilon^{1+\alpha}\right. \\
& \quad-\frac{\left(a_{n}^{2} t+b_{n}\left(t^{2}+t\right) \varepsilon+\bar{\varphi}_{n}(t, 1) \varepsilon^{1+\alpha}\right)\left(a_{n}^{2} s+b_{n}\left(s+s^{2}\right) \varepsilon+\bar{\varphi}_{n}(1, s) \varepsilon^{1+\alpha}\right)}{a_{n}^{2}+2 b_{n} \varepsilon+\bar{\varphi}_{n}(1,1) \varepsilon^{1+\alpha}+\mathcal{R}_{\varepsilon}^{1}(1,1)} \\
& \left.\quad+\mathcal{R}_{\varepsilon}^{1}(t, s)\right) \\
& \quad a_{n}^{2} \varepsilon^{2} \\
\quad & \quad \times\left(\left(\bar{\varphi}_{n}(t, s)+t s \bar{\varphi}_{n}(1,1)-t \bar{\varphi}_{n}(1, s)-s \bar{\varphi}_{n}(t, 1)\right) \varepsilon^{1+\alpha}\right. \\
\quad & \left.\quad b_{n}^{2}\left(t s^{2}+t^{2} s-t^{2} s^{2}-s t\right) \varepsilon^{2}+\mathcal{R}_{\varepsilon}^{1}(t, s)\right) .
\end{aligned}
$$

Therefore, the theorem holds.

Let us observe that if the covariance function $k(t, s)$ is more regular, that is, $C^{4+\beta}$ for some $\beta \geq 0$, then Theorem 4.3 continues to hold and the associated asymptotic covariance $\bar{k}_{Y}$, given by (4.10), is not in general degenerate. In fact, since the fourth derivatives exist, we obtain $\bar{\varphi}_{n}(t, s)=e_{n}\left(t^{3} s+t s^{3}\right)+f_{n} t^{2} s^{2}$, where $e_{n}$ and $f_{n}$ are suitable constants. Therefore, tedious but straightforward computations will give $\bar{k}_{Y}(t, s)=($ constant $) t s(1-t)(1-s)$.

Let us now return to Example 4.2 which dealt with our first result for the bridge of the $n$-fold conditional process, that is, the following integrated Gaussian process:

$$
X_{t}=\int_{0}^{t} Z_{u} \mathrm{~d} u
$$

where $Z$ is a centered Gaussian process with covariance function $\kappa(t, s)$. We are looking for conditions on $\kappa$ so that Assumption 4.1 or 4.2 is satisfied and then a large deviation principle as in Theorem 4.2 or 4.3 holds. We have the following proposition. 
Proposition 4.1. (i) Suppose that, for some $\alpha \in(0,1]$,

$$
\begin{gathered}
\kappa\left(T_{n}+\varepsilon u, T_{n}+\varepsilon v\right)=\kappa\left(T_{n}, T_{n}\right)+\varepsilon^{\alpha} \hat{g}(u, v)+\hat{\mathcal{R}}_{\varepsilon}(u, v), \\
\int_{0}^{T} \kappa\left(T_{n}+\varepsilon u, v\right) \mathrm{d} v=\int_{0}^{T} \mathrm{~d} v \kappa\left(T_{n}, v\right)+\varepsilon^{\alpha} \tilde{g}(u ; T)+\tilde{\mathcal{R}}_{\varepsilon}(u ; T), \quad T>0,
\end{gathered}
$$

(the above functions and remaining terms may all depend on $\left.T_{n}\right)$ with $\hat{g} \in L^{1}\left([0,1]^{2}\right)$, $\tilde{g}(\cdot ; T) \in L^{1}([0,1])$, and

$$
\lim _{\varepsilon \rightarrow 0} \varepsilon^{-\alpha}\left\|\hat{\mathcal{R}}_{\varepsilon}(\cdot, \cdot)\right\|_{L^{1}\left([0,1]^{2}\right)}=0 \quad \text { and } \quad \lim _{\varepsilon \rightarrow 0} \varepsilon^{-\alpha}\left\|\tilde{\mathcal{R}}_{\varepsilon}(\cdot ; T)\right\|_{L^{1}([0,1])}=0 .
$$

Then, Assumption 4.1 holds with

$$
\bar{\varphi}(t, s)=\int_{0}^{t} \mathrm{~d} u \int_{0}^{s} \mathrm{~d} v \hat{g}(u, v) \text { and } \bar{\psi}(t, T)=\int_{0}^{t} \mathrm{~d} u \tilde{g}(u ; T) .
$$

(ii) Suppose that, for some $\alpha \in(0,1]$,

$$
\begin{gathered}
\kappa\left(T_{n}+\varepsilon u, T_{n}+\varepsilon v\right)=\kappa\left(T_{n}, T_{n}\right)+\varepsilon e(u+v)+\varepsilon^{1+\alpha} \hat{g}(u, v)+\hat{\mathcal{R}}_{\varepsilon}(u, v), \\
\int_{0}^{T} \kappa\left(T_{n}+\varepsilon u, v\right) \mathrm{d} v=\int_{0}^{T} \mathrm{~d} v \kappa\left(T_{n}, v\right)+\varepsilon u f(T)+\varepsilon^{1+\alpha} \tilde{g}(u ; T)+\tilde{\mathcal{R}}_{\varepsilon}(u ; T), \quad T>0,
\end{gathered}
$$

(the above functions, remaining terms, and constants $e$ and $f(T)$ may all depend on $T_{n}$ ), with $\hat{g} \in L^{1}\left([0,1]^{2}\right), \tilde{g}(\cdot ; T) \in L^{1}([0,1])$, and

$$
\lim _{\varepsilon \rightarrow 0} \varepsilon^{-(1+\alpha)}\left\|\hat{\mathcal{R}}_{\varepsilon}(\cdot, \cdot)\right\|_{L^{1}\left([0,1]^{2}\right)}=0 \quad \text { and } \quad \lim _{\varepsilon \rightarrow 0} \varepsilon^{-(1+\alpha)}\left\|\tilde{\mathcal{R}}_{\varepsilon}(\cdot ; T)\right\|_{L^{1}([0,1])}=0 .
$$

Then, Assumption 4.2 holds with

$$
\bar{\varphi}(t, s)=\int_{0}^{t} \mathrm{~d} u \int_{0}^{s} \mathrm{~d} v \hat{g}(u, v) \text { and } \bar{\psi}(t, T)=\int_{0}^{t} \mathrm{~d} u \tilde{g}(u ; T) .
$$

The proof is straightforward and postponed to Appendix B.

Example 4.4. (m-fold integrated Brownian motion.) Let us return to Example 4.3 with $X$ as the following $m$-fold integrated Brownian motion:

$$
X_{t}=\int_{0}^{t} \mathrm{~d} u\left(\int_{0}^{u} \mathrm{~d} u_{m-1} \cdots \int_{0}^{u_{2}} \mathrm{~d} u_{1} W_{u_{1}}\right),
$$

where $W$ denotes a standard Brownian motion. Recall that here the covariance function is

$$
k(t, s)=\int_{0}^{t} \int_{0}^{s} \kappa(u, v) \mathrm{d} u \mathrm{~d} v \quad \text { with } \kappa(t, s)=\frac{1}{((m-1) !)^{2}} \int_{0}^{t \wedge s}(t-\xi)^{m-1}(s-\xi)^{m-1} \mathrm{~d} \xi .
$$

Things are slightly different according to whether $m=1$ or $m \geq 2$. Let us consider the $m \geq 2$ case; the case in which $m=1$ is dealt with in Example 4.5, below. Straightforward computations allow us to show that

$$
\begin{aligned}
\kappa\left(T_{n}+\varepsilon u, T_{n}+\varepsilon v\right)= & \kappa\left(T_{n}, T_{n}\right)+\varepsilon \frac{1}{2} T_{n}^{2 m-2}(u+v) \\
& +\varepsilon^{2} \frac{(m-1)}{2 m-3} T_{n}^{2 m-3}\left(\frac{(m-2)}{2}(u+v)^{2}+u v\right)+\mathcal{O}\left(\varepsilon^{3}\right)
\end{aligned}
$$


and

$$
\begin{aligned}
\int_{0}^{T} \kappa\left(T_{n}+\varepsilon u, v\right) \mathrm{d} v= & \int_{0}^{T} \kappa\left(T_{n}, v\right) \mathrm{d} v+\varepsilon u \frac{m-1}{m} \int_{0}^{T}\left(T_{n}-x\right)^{m-2}(T-x)^{m} \mathrm{~d} x \\
& +\varepsilon^{2} u^{2} \frac{(m-2)(m-1)}{m} \int_{0}^{T}\left(T_{n}-x\right)^{m-3}(T-x)^{m} \mathrm{~d} x+\mathcal{O}\left(\varepsilon^{3}\right),
\end{aligned}
$$

in which $\mathcal{O}\left(\varepsilon^{3}\right)$ denotes a function going to 0 as $\varepsilon \rightarrow 0$ in the right $L^{1}$ space at speed $\varepsilon^{3}$. Therefore, thanks to (4.11), (4.12), and Proposition 4.1, Assumption 4.2 does hold with

$$
\begin{gathered}
\bar{\varphi}(t, s)=\frac{(m-1)}{4(2 m-3)} T_{n}^{2 m-3}\left((m-2)\left(t^{2} s+t s^{2}\right)+t^{2} s^{2}\right), \\
\bar{\psi}(t, T)=\left(\frac{1}{2} \frac{(m-2)(m-1)}{m} \int_{0}^{T}\left(T_{n}-x\right)^{m-3}(T-x)^{m} \mathrm{~d} x\right) t^{2} .
\end{gathered}
$$

By using Theorem 4.3, we can assert that the bridge process $Y$ satisfies a (nondegenerate) large deviation principle with inverse speed $\varepsilon^{4}$ and asymptotic covariance as in (4.10) with $\alpha=1$.

Example 4.5. (Integrated fractional Brownian motion.) Let $X_{t}=\int_{0}^{t} Z_{u} \mathrm{~d} u$, where $Z$ is a fractional Brownian motion with Hurst index $H$. This is quite an interesting example because, according to whether $H \leq \frac{1}{2}$ or $H>\frac{1}{2}$, we obtain both cases studied in Proposition 4.1. In fact, straightforward computations allow us to state that

$$
\kappa_{H}\left(T_{n}+\varepsilon u, T_{n}+\varepsilon v\right)=\kappa_{H}\left(T_{n}, T_{n}\right)+H T_{n}^{2 H-1}(u+v) \varepsilon-\frac{1}{2}|u-v|^{2 H} \varepsilon^{2 H}+\mathcal{O}\left(\varepsilon^{2}\right)
$$

and

$$
\begin{aligned}
& \int_{0}^{T} \kappa_{H}\left(T_{n}+\varepsilon u, v\right) \mathrm{d} v
\end{aligned}
$$

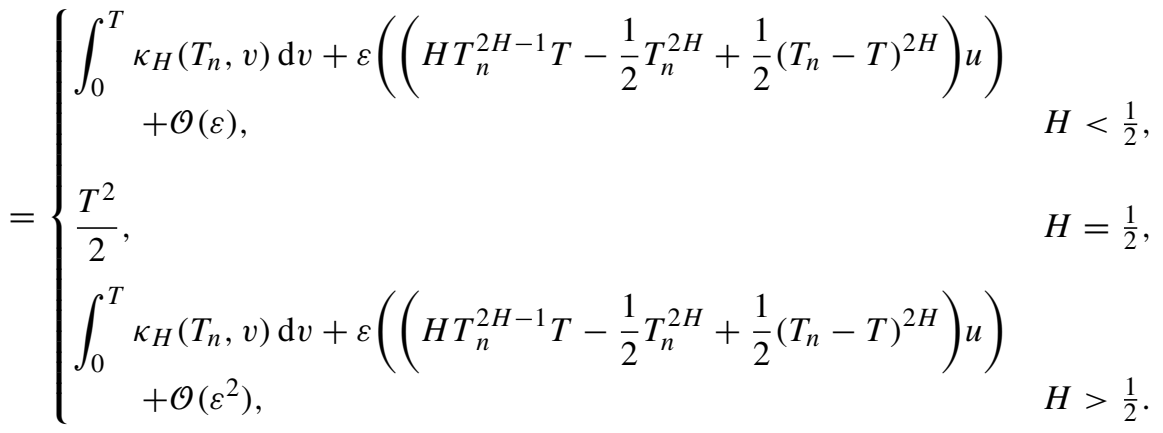

Therefore, the asymptotic behavior can be resumed as follows.

(a) If $H<\frac{1}{2}$, Proposition 4.1(i) holds with $\alpha=2 H$ and

$$
\hat{g}\left(u, v ; T_{n}\right)=-\frac{1}{2}|u-v|^{2 H}, \quad \tilde{g}\left(u ; T_{n}, T\right) \equiv 0 .
$$

(b) If $H=\frac{1}{2}$, Proposition 4.1(i) holds again with $\alpha=1$ and

$$
\hat{g}\left(u, v ; T_{n}\right)=\frac{1}{2}((u+v)-|u-v|)=u \wedge v, \quad \tilde{g}\left(u ; T_{n}, T\right) \equiv 0 .
$$


(c) If $H>\frac{1}{2}$, Proposition 4.1(ii) holds with $\alpha=2 H-1$ and

$$
\hat{g}\left(u, v ; T_{n}\right)=-\frac{1}{2}|u-v|^{2 H}, \quad \tilde{g}\left(u ; T_{n}, T\right)=0 .
$$

In conclusion, by suitably applying Theorems 4.2 and 4.3 , the family of bridges $\left(Y_{T_{n}+\varepsilon}^{n}\right)_{\varepsilon}$ satisfies a large deviation principle on $C([0,1])$ with inverse speed $\varepsilon^{2+2 H}$ and asymptotic covariance function given by

$$
\bar{k}_{Y}(t, s)=\bar{\varphi}_{H}(t, s)+t s \bar{\varphi}_{H}(1,1)-t \bar{\varphi}_{H}(1, s)-s \bar{\varphi}_{H}(t, 1),
$$

where

$$
\bar{\varphi}_{H}(t, s) \equiv \bar{\varphi}_{n}(t, s)= \begin{cases}\frac{\left(|t-s|^{2 H+2}-t^{2 H+2}-s^{2 H+2}\right)}{2(2 H+1)(2 H+2)}, & H \neq \frac{1}{2}, \\ \frac{(t \wedge s)^{3}}{3}+\frac{(t \wedge s)^{2}}{2}|t-s|, & H=\frac{1}{2} .\end{cases}
$$

Let us add some further remarks. In the case in which $H=\frac{1}{2}$, it is immediate to check that $\bar{\varphi}_{H}(t, s)=\int_{0}^{t} \int_{0}^{s} \kappa_{H}(u, v) \mathrm{d} u \mathrm{~d} v$. In other words, $\bar{\varphi}_{1 / 2}$ turns out to be the covariance function of the process $X$. Then, by taking into account Remark 4.2, the large deviations associated to the bridge of the $n$-fold integrated Brownian motion behave as 'the false bridge', even if with a faster speed (in fact, in this case the inverse speed is $\varepsilon^{3}$, while the inverse speed of the nonconditioned $n$-fold process is given by $\varepsilon^{2}$ ).

\section{The asymptotic behavior of the crossing probability}

In this section previous results are applied in order to state the large deviation asymptotic behavior of the hitting probability, the underlying process of interest being the bridge of an $n$-fold conditional Gaussian process. The already collected results can be resumed in the following hypothesis.

Hypothesis 5.1. 1. The family of $n$-fold conditional processes $\left\{\left(X_{T_{n}+\varepsilon t}^{n}\right)_{t \in[0,1]}\right\}_{\varepsilon}$ satisfies a large deviation principle with inverse speed $\gamma_{\varepsilon}^{2}$ and rate function

$$
J_{n}(h)= \begin{cases}\frac{1}{2}\left\|h-x_{n}\right\|_{\overline{\mathscr{H}}_{n}}^{2} & \text { if } h_{0}=x_{n} \text { and } h-\bar{m} \in \overline{\mathscr{H}}_{n}, \\ +\infty & \text { otherwise }\end{cases}
$$

where $\overline{\mathscr{H}}_{n}$ is the reproducing kernel Hilbert space associated to a suitable covariance function $\bar{k}_{n}$.

2. The family of bridges of the $n$-fold conditional processes $\left\{\left(Y_{T_{n}+\varepsilon t}^{n}\right)_{t \in[0,1]}\right\}_{\varepsilon}$ satisfies a large deviation principle with inverse speed $\eta_{\varepsilon}^{2}$ and rate function

$$
J_{Y}(h)= \begin{cases}\frac{1}{2}\|h-\bar{m}\|_{\overline{\mathscr{H}}_{Y}}^{2} & \text { if } h_{0}=x_{n}, h_{1}=y, \text { and } h-\bar{m} \in \overline{\mathscr{H}}_{Y}, \\ +\infty & \text { otherwise, }\end{cases}
$$

where $\overline{\mathscr{H}}_{Y}$ is the reproducing kernel Hilbert space associated to a suitable covariance function $\bar{k}_{Y}$ and $\bar{m}_{t}=x_{n}+\bar{\beta}_{t}\left(y-x_{n}\right) \equiv x_{n}+\bar{k}_{n}(t, 1)\left(y-x_{n}\right) / \bar{k}_{n}(1,1)$.

Throughout this section, we assume that Hypothesis 5.1 always holds.

Now, let us first focus on the upper barrier case, the same arguments will apply for lower barriers. 
Let $U: \mathbb{R} \rightarrow \mathbb{R}$ be a continuous function standing for an upper barrier, and consider the probability that $Y_{T_{n}+\varepsilon}^{n}$. reaches the barrier $U$ up to the final time 1 , that is,

$$
\mathrm{P}\left(\tau_{\varepsilon}^{U} \leq 1\right) \quad \text { with } \tau_{\varepsilon}^{U}=\inf \left\{t>0: Y_{T_{n}+\varepsilon t}^{n} \geq U_{T_{n}+\varepsilon t}\right\} .
$$

The above probability is negligible if $Y_{T_{n}}^{n}=x_{n}<U_{T_{n}}$ and $Y_{T_{n}+\varepsilon t}^{n}=y<U_{T_{n}+\varepsilon t}$ for any $\varepsilon$ close to 0 , that is, $y \leq U_{T_{n}}$. As we will see, the case in which $y=U_{T_{n}}$ will give a nonrelevant estimate, so that we can assume that both $x_{n}$ and $y$ are less than $U_{T_{n}}$. So, if $x_{n}, y<U_{T_{n}}$, we have

$$
\lim _{\varepsilon \rightarrow 0} \eta_{\varepsilon}^{2} \log \mathrm{P}\left(\tau_{\varepsilon}^{U} \leq 1\right)=-I_{Y}^{U},
$$

with $I_{Y}^{U}>0$. Let us now see what form $I_{Y}^{U}$ takes. Set $Z_{T_{n}+\varepsilon t}^{n}=Y_{T_{n}+\varepsilon t}^{n}-U_{T_{n}+\varepsilon t}$. Since $\lim _{\varepsilon \rightarrow 0} U_{T_{n}+\varepsilon t}=U_{T_{n}}$ uniformly for $t \in[0,1]$, by contraction, it immediately follows that $\left\{\left(Z_{T_{n}+\varepsilon t}^{n}\right)_{t \in[0,1]}\right\}_{\varepsilon}$ satisfies a large deviation principle as well, with the same inverse speed and rate function

$$
J_{Z}(h)=J_{Y}\left(h+U_{T_{n}}\right)
$$

Then, we have

$$
\lim _{\varepsilon \rightarrow 0} \eta_{\varepsilon}^{2} \log \mathrm{P}\left(\tau_{\varepsilon}^{U} \leq 1\right)=-\inf _{\gamma \in \Gamma_{U}} J_{Y}\left(\gamma+U_{T_{n}}\right)=-I_{Y}^{U}
$$

where $\Gamma_{U}=\left\{\gamma: \sup _{t \in[0,1]} \gamma_{t} \geq 0\right\}$.

If a (continuous) lower barrier $L_{t}$ was considered then the same arguments would apply, giving

$$
\lim _{\varepsilon \rightarrow 0} \eta_{\varepsilon}^{2} \log \mathrm{P}\left(\tau_{\varepsilon}^{L} \leq 1\right)=-\inf _{\gamma \in \Gamma_{L}} J_{Y}\left(\gamma+L_{T_{n}}\right)=-I_{Y}^{L},
$$

where $\tau_{\varepsilon}^{L}=\inf \left\{t>0: Y_{T_{n}+\varepsilon t}^{n} \leq L_{T_{n}+\varepsilon t}\right\}$ and $\Gamma_{L}=\left\{\gamma: \inf _{t \in[0,1]} \gamma_{t} \leq 0\right\}$, and this is interesting when $x_{n}, y>L_{T_{n}}$. Finally, in the double barrier case, with $L_{t} \leq U_{t}$ for any $t$, the hitting probability behaves as follows:

$$
\lim _{\varepsilon \rightarrow 0} \eta_{\varepsilon}^{2} \log \mathrm{P}\left(\tau_{\varepsilon}^{L, U} \leq 1\right)=-I_{Y}^{L, U},
$$

where $\tau_{\varepsilon}^{L, U}=\tau_{\varepsilon}^{L} \wedge \tau_{\varepsilon}^{U}$ is the first time at which $Y_{T_{n}+\varepsilon}^{n}$. reaches at least one barrier and $I_{Y}^{L, U}$ is a suitable quantity, which is strictly positive if $x_{n}, y \in\left(L_{T_{n}}, U_{T_{n}}\right)$.

The quantities $I_{Y}^{U}, I_{Y}^{L}$, and $I_{Y}^{L, U}$ are computed in the next proposition.

Proposition 5.1. Suppose that $L$ and $U$ are continuous functions with $L_{t} \leq U_{t}$ for any $t \in$ [0,1]. Then,

$$
\begin{gathered}
I_{Y}^{U}=\inf _{t \in[0,1]} \frac{\left(\left(U_{T_{n}}-x_{n}\right)\left(1-\bar{\beta}_{t}\right)+\bar{\beta}_{t}\left(U_{T_{n}}-y\right)\right)^{2}}{2 \bar{k}_{Y}(t, t)} \quad \text { if } x_{n}, y<U_{T_{n}}, \\
I_{Y}^{L}=\inf _{t \in[0,1]} \frac{\left(\left(x_{n}-L_{T_{n}}\right)\left(1-\bar{\beta}_{t}\right)+\bar{\beta}_{t}\left(y-L_{T_{n}}\right)\right)^{2}}{2 \bar{k}_{Y}(t, t)} \text { if } x_{n}, y>L_{T_{n}}, \\
I_{Y}^{L, U}=\min \left(I_{Y}^{L}, I_{Y}^{U}\right) \quad \text { if } x_{n}, y \in\left(L_{T_{n}}, U_{T_{n}}\right) .
\end{gathered}
$$

Proof. Consider the first equality (single upper barrier case). We have to show that

$$
\inf _{\gamma \in \hat{\Gamma}_{U}} \frac{1}{2}\left\|\gamma+U_{T_{n}}-\bar{m}\right\|_{\overline{\mathcal{H}}_{Y}}^{2}=\inf _{t \in[0,1]} \frac{\left(\left(U_{T_{n}}-x_{n}\right)\left(1-\bar{\beta}_{t}\right)+\bar{\beta}_{t}\left(U_{T_{n}}-y\right)\right)^{2}}{2 \bar{k}_{Y}(t, t)},
$$


where $\hat{\Gamma}_{U}=\left\{\gamma: \gamma+U_{T_{n}}-\bar{m} \in \overline{\mathscr{H}}_{Y}, \sup _{t \in[0,1]} \gamma_{t} \geq 0\right\}$. Setting $\hat{\Gamma}_{t, U}=\left\{\gamma: \gamma+U_{T_{n}}-\bar{m} \in\right.$ $\left.\overline{\mathcal{H}}_{Y}, \gamma_{t}=0\right\}$, we have $\hat{\Gamma}=\bigcup_{0<t<1} \hat{\Gamma}_{t}$, so that we simply need

$$
\inf _{\gamma \in \hat{\Gamma}_{t, U}} \frac{1}{2}\left\|\gamma+U_{T_{n}}-\bar{m}\right\|_{\overline{\mathscr{H}}_{Y}}^{2}=\frac{\left(\left(U_{T_{n}}-x_{n}\right)\left(1-\bar{\beta}_{t}\right)+\bar{\beta}_{t}\left(U_{T_{n}}-y\right)\right)^{2}}{2 \bar{k}_{Y}(t, t)} .
$$

As already seen (see Section 2), a set of paths which is dense in $\overline{\mathscr{H}}_{Y}$ is the one formed by those which are the barycenters of the RV belonging to the dual space of $C([0,1])$, that is,

$$
\gamma_{u}+U_{T_{n}}-\bar{m}_{u}=\int_{0}^{1} \bar{k}_{Y}(u, v) \lambda(\mathrm{d} v)
$$

as $\lambda$ varies in $\mathcal{M}[0,1]$. Since, for such kind of paths,

$$
\left\|\gamma+U_{T_{n}}-\bar{m}\right\|_{\overline{\mathcal{H}}_{Y}}^{2}=\int_{0}^{1} \int_{0}^{1} \bar{k}_{Y}(u, v) \lambda(\mathrm{d} u) \lambda(\mathrm{d} v),
$$

it is enough to minimize the right-hand side of the above equation with respect to $\lambda$, with the additional constraint that $\gamma_{t}=0$, which yields

$$
\bar{m}_{t}-U_{T_{n}}+\int_{0}^{1} \bar{k}_{Y}(t, v) \lambda(\mathrm{d} v)=0 .
$$

This is a constrained extremum problem: using Lagrange multipliers, $\lambda$ must satisfy

$$
\int_{0}^{1} \bar{k}_{Y}(u, v) \lambda(\mathrm{d} v)-\alpha \bar{k}_{Y}(t, u)=0 \quad \text { for any } u \in[0,1]
$$

for some $\alpha \in \mathbb{R}$. Taking care of the constraint, we find that

$$
\alpha=\frac{U_{T_{n}}-\bar{m}_{t}}{\bar{k}_{Y}(t, t)}, \quad \lambda(\mathrm{d} v)=\frac{U_{T_{n}}-\bar{m}_{t}}{\bar{k}_{Y}(t, t)} \delta_{\{t\}}(\mathrm{d} v),
$$

where $\delta_{\{t\}}$ stands for the Dirac mass in $t$. Therefore,

$$
\inf _{w \in \hat{\Gamma}_{t, U}} \frac{1}{2} \int_{0}^{1} \int_{0}^{1} \bar{k}_{Y}(u, v) \lambda(u) \lambda(\mathrm{d} v)=\frac{\left(U_{T_{n}}-\bar{m}_{t}\right)^{2}}{2 \bar{k}_{Y}(t, t)},
$$

and the statement immediately follows by recalling that $\bar{m}_{t}=x_{n}+\bar{\beta}_{t}\left(y-x_{n}\right)$.

Concerning the second equality, it follows by developing analogous arguments. As for the final equality, it is standard in large deviation theory (see, e.g. the discussion in the proof of Theorem 2.2 of [3]).

Let us stress that the barriers $U$ and/or $L$ can also be piecewise continuous, in which case the previous machinery runs again if the jump times coincide with some of the conditional times $T_{1}, \ldots, T_{n}$.

Before we develop some examples, let us recall that $\bar{\beta}_{t}=\bar{k}_{n}(t, 1) / \bar{k}_{n}(1,1)$, where $\bar{k}_{n}$ is as defined in (3.12) and represents the asymptotic covariance function associated to the $n$-fold conditional process. When our first set of large deviation results for the bridge holds (as in Subsection 4.1), we have

$$
\bar{k}_{Y}(t, s)=\bar{k}_{n}(t, s)-\frac{\bar{k}_{n}(t, 1) \bar{k}_{n}(s, 1)}{\bar{k}_{n}(1,1)},
$$


with $\bar{k}_{Y}$ being more complicated if it takes a more complicated form, as seen in Subsection 4.2. Therefore, the minimization problem, as required to compute $I_{Y}^{U}$ and $I_{Y}^{L}$, does not have a closed form in general; so, for practical purposes, we might be forced to use some numerical method (e.g. the Newton method).

Example 5.1. (Integrated Brownian motion.) Following Example 4.5 (with $H=\frac{1}{2}$ ), let us consider $X_{t}=\int_{0}^{t} B_{s} \mathrm{~d} s$, where $B$ is a standard Brownian motion. Such a process has interesting applications in metrology, where it is used as a model for the atomic clock error and the exit from a fixed boundary means that the clock error exceeds an allowed limit and so must be resynchronized. Here, we are in the second set of our large deviation estimates: the bridge of the $n$-fold conditional process satisfies a large deviation principle at inverse speed $\varepsilon^{3}$ and with rate function associated to the asymptotic covariance function

$$
\bar{k}_{Y}(t, s)=\bar{\varphi}(t, s)+t s \bar{\varphi}(1,1)-t \bar{\varphi}(1, s)-s \bar{\varphi}(t, 1)
$$

with

$$
\bar{\varphi}(t, s)=\frac{(t \wedge s)^{3}}{3}+\frac{(t \wedge s)^{2}}{2}|t-s| .
$$

Since $\bar{k}_{n}(t, s)=a_{n}^{2} t s$, we have $\bar{\beta}_{t}=t$, so that $I_{Y}^{U}=g\left(U_{T_{n}}\right)$ and $I_{Y}^{L}=g\left(L_{T_{n}}\right)$ with

$$
g(a)=\inf _{t \in[0,1]} \frac{\left(\left|a-x_{n}\right|(1-t)+t|a-y|\right)^{2}}{2 t^{2}(1-t)^{2} / 3} .
$$

The solution is simple to find:

$$
g(a)=\frac{3}{2}\left(\left|a-x_{n}\right|^{1 / 2}+|a-y|^{1 / 2}\right)^{4} .
$$

Example 5.2. (Fractional Brownian motion.) Following Subsection 3.1 and Example 4.1, let us consider a fractional Brownian motion $X$ with Hurst index $H$, in which we have

$$
\bar{k}_{n}(t, s)=k_{H}(t, s)=\frac{t^{2 H}+s^{2 H}-|t-s|^{2 H}}{2} .
$$

So, in order to compute $I_{Y}^{U}$ and $I_{Y}^{L}$, giving the asymptotic behavior of the hitting probability of the bridge $Y^{n}$, by Proposition 5.1 we should be able to compute

$$
g_{H}(a)=\inf _{t \in[0,1]} \frac{\left(\left(a-x_{n}\right)\left(1-k_{H}(t, 1)\right)+k_{H}(t, 1)(a-y)\right)^{2}}{2\left(k_{H}(t, t)-k_{H}^{2}(t, 1)\right)},
$$

either with $a>x_{n}, y$ or $a<x_{n}, y$. In fact, we have $I_{Y}^{U}=g_{H}\left(U_{T_{n}}\right)$ and $I_{Y}^{L}=g_{H}\left(L_{T_{n}}\right)$. As far as we know, the exact solution can be computed only when $H=\frac{1}{2}$, that is, when a standard Brownian motion is taken into account, in which case we have

$$
g_{1 / 2}(a)=2\left(a-x_{n}\right)(a-y),
$$

which agrees with well-known formulae (see, e.g. [2]).

In relation to Example 5.2, we have performed some numerical experiments concerning the fractional Brownian motion. In particular, we have estimated via Monte Carlo methods the probability of crossing the upper barrier $U=1$ up to time 1 in two different ways: by crude simulations, in which the exit is reached if a simulated position is greater than $U=1$, and by 
TABLE 1: Fractional Brownian motion: Monte Carlo estimated probability of crossing the upper barrier $U=1$ up to time 1 for varying values of the Hurst index $H$. The associated $95 \%$ confidence interval is given in the brackets.

\begin{tabular}{lcccc}
\hline Method & Step size & $H=0.3$ & $H=0.5$ & $H=0.7$ \\
\hline Corrected & 0.010 & 0.60876 & 0.31820 & 0.20564 \\
& & $(0.60573,0.61178)$ & $(0.31531,0.32109)$ & $(0.20313,0.20814)$ \\
Corrected & 0.002 & 0.61841 & 0.31980 & 0.20274 \\
& & $(0.61540,0.62142)$ & $(0.31691,0.32269)$ & $(0.20025,0.20523)$ \\
Crude & 0.010 & 0.47909 & 0.28918 & 0.19884 \\
& & $(0.47599,0.48219)$ & $(0.28637,0.29199)$ & $(0.19637,0.20131)$ \\
Crude & 0.002 & 0.54114 & 0.30496 & 0.20222 \\
& & $(0.53805,0.54423)$ & $(0.30211,0.30781)$ & $(0.19973,0.20471)$ \\
Crude & 0.001 & 0.56082 & 0.30878 & 0.20251 \\
& & $(0.55774,0.56390)$ & $(0.30592,0.31164)$ & $(0.20002,0.20500)$ \\
\hline
\end{tabular}

means of the corrected procedure, as recalled in the introduction, for which at each step the crossing is decided by using the large deviation approximation for the exit probability of the pinned process. In all the experiments the exit probability is numerically computed through $10^{5}$ simulations. The results are given in terms of the method (corrected or crude) and of the step size $(\varepsilon=0.01,0.002,0.001)$ for varying values of the Hurst index $H$, which is set equal to 0.3 , 0.5 , and 0.7 . Whenever $H=0.5$, everything is known (exit probability is equal to 0.31732 ), including the fact that the crude approach works very poorly, so it has been considered to assess the procedure and for comparison purposes. The choices $H=0.3$ and $H=0.7$ have been taken into account to compare the results when $H<0.5$ (short memory, more irregular paths) and $H>0.5$ (long memory, less irregular paths). The results, given in Table 1, show the level of sensitivity with respect to the method (corrected or crude) when $H$ decreases, that is, when the irregularity of the path tends to be higher. This is not surprising because the inverse speed of the large deviations for the bridge is in fact $\varepsilon^{2 H}$, so that the correction works more when $H$ decreases.

Let us give a final remark. Our simulation scheme relies on the assumption that the past pinned instants are fixed, although they are all of order $\varepsilon$, and only the step size of the current time interval is considered negligible. In fact, the past observations have been considered as a datum, that is, fixed, and only the bridge's length is supposed to be small enough to approximate the exit probability with its large deviation estimate. As remarked by an anonymous referee, it would be interesting to see what happens when the size of all the past time intervals goes to 0 , a case in which the 'local independence' fails. We are now working with this case and it will be interesting to measure the sensitivity of the method with respect to the independence of the past, by comparing the new results with the ones reported in Table 1.

\section{Appendix A. Proof of (4.4)}

Let $J_{Y}$ be the rate function given by Theorem 4.1, i.e.

$$
J_{Y}(h)= \begin{cases}\frac{1}{2}\|h-\bar{m}\|_{\overline{\mathscr{H}}_{Y}}^{2} & \text { if } h_{0}=x_{n}, h_{1}=y, \text { and } h-\bar{m} \in \overline{\mathscr{H}}_{Y}, \\ +\infty & \text { otherwise }\end{cases}
$$


where $\bar{m}_{t}=x_{n}+\bar{\beta}_{t}\left(y-x_{n}\right)$ and $\overline{\mathscr{H}}_{Y}$ is the reproducing kernel Hilbert space associated to the covariance function

$$
\bar{k}_{Y}(t, s)=\bar{k}_{n}(t, s)-\bar{\beta}_{s} \bar{k}_{n}(t, 1)=\bar{k}_{n}(t, s)-\frac{\bar{k}_{n}(t, 1) \bar{k}_{n}(s, 1)}{\bar{k}_{n}(1,1)},
$$

where $\bar{k}_{n}$ is as defined in (3.12). We claim that $J_{Y}$ can be written as

$$
J_{Y}(h)= \begin{cases}\frac{1}{2}\left(\left\|h-x_{n}\right\|_{\overline{\mathcal{H}}_{n}}^{2}-\frac{\left(y-x_{n}\right)^{2}}{\bar{k}_{n}(1,1)}\right) & \text { if } h_{0}=x_{n}, h_{1}=y, \text { and } h-x_{n} \in \overline{\mathscr{H}}_{n}, \\ +\infty & \text { otherwise }\end{cases}
$$

where $\overline{\mathscr{H}}_{n}$ is the reproducing kernel Hilbert space associated to the covariance function $\bar{k}_{n}$. Let us observe that this can be done in two ways: by large deviation arguments (in particular, by using contraction type properties that allow for the transfer of large deviation principles) or by handling reproducing kernel Hilbert spaces. Here, we follow the second way.

First, let us prove that the sets where the two functionals are finite are the same, that is, $\mathcal{K}_{1}=\mathcal{K}_{2}$, being

$$
\begin{aligned}
& \mathcal{K}_{1}=\left\{h: h_{0}=x_{n}, h_{1}=y, h-\bar{m} \in \overline{\mathscr{H}}_{Y}\right\}, \\
& \mathcal{K}_{2}=\left\{h: h_{0}=x_{n}, h_{1}=y, h-\bar{m} \in \overline{\mathscr{H}}_{n}\right\} .
\end{aligned}
$$

If we set

$$
\begin{aligned}
& \mathcal{D}_{1}:=\left\{h \in \mathcal{K}_{1}: h_{t}-\bar{m}_{t}=\int_{0}^{1} \bar{k}_{Y}(t, s) \alpha(\mathrm{d} s) \text { for some } \alpha \in \mathcal{M}[0,1]\right\}, \\
& \mathcal{D}_{2}:=\left\{h \in \mathcal{K}_{2}: h_{t}-\bar{m}_{t}=\int_{0}^{1} \bar{k}_{h}(t, s) \gamma(\mathrm{d} s) \text { for some } \gamma \in \mathcal{M}[0,1]\right\},
\end{aligned}
$$

then the statement would follow from

$$
\mathscr{D}_{1}=\mathscr{D}_{2} \quad \text { and } \quad\|h-\bar{m}\|_{\overline{\mathscr{H}}_{Y}}=\|h-\bar{m}\|_{\overline{\mathcal{H}}_{n}} \quad \text { for any } h \in \mathscr{D}_{1}=\mathscr{D}_{2} .
$$

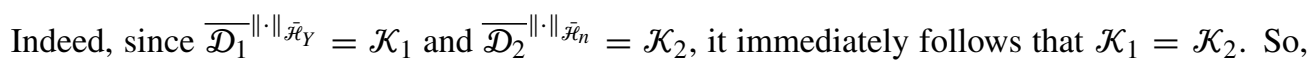
let us show that (A.1) does hold.

If we take $h \in \mathscr{D}_{1}$ then

$$
\begin{aligned}
h_{t}-\bar{m}_{t} & =\int_{0}^{1} \bar{k}_{Y}(t, s) \alpha(\mathrm{d} s) \\
& =\int_{0}^{1}\left(\bar{k}_{n}(t, s)-\frac{\bar{k}_{n}(1, t) \bar{k}_{n}(1, s)}{\bar{k}_{n}(1,1)}\right) \alpha(\mathrm{d} s) \\
& =\int_{0}^{1} \bar{k}_{n}(t, s)\left(\alpha(\mathrm{d} s)-\frac{\int_{0}^{1} \bar{k}_{n}(1, u) \alpha(\mathrm{d} u)}{\bar{k}_{n}(1,1)} \delta_{\{1\}}(\mathrm{d} s)\right),
\end{aligned}
$$

where $\delta_{\{1\}}$ denotes the Dirac mass, and then $h \in \mathscr{D}_{2}$. Conversely, if $h \in \mathscr{D}_{2}$ then $h_{t}-\bar{m}_{t}=$ $\int_{0}^{1} \bar{k}_{n}(t, s) \gamma(\mathrm{d} s)$ and, in particular, it must be

$$
0=h_{1}-\bar{m}_{1}=\int_{0}^{1} \bar{k}_{n}(1, s) \gamma(\mathrm{d} s) .
$$


Therefore,

$$
\begin{aligned}
h_{t}-\bar{m}_{t} & =\int_{0}^{1} \bar{k}_{n}(t, s) \gamma(\mathrm{d} s) \\
& =\int_{0}^{1}\left(\bar{k}_{n}(t, s)-\frac{\bar{k}_{n}(1, t) \bar{k}_{n}(1, s)}{\bar{k}_{n}(1,1)}\right) \gamma(\mathrm{d} s) \\
& =\int_{0}^{1} \bar{k}_{Y}(t, s) \gamma(\mathrm{d} s)
\end{aligned}
$$

and $h \in \mathscr{D}_{1}$. Finally,

$$
\|h-\bar{m}\|_{\overline{\mathscr{H}}_{Y}}^{2}=\int_{0}^{1} \int_{0}^{1} \bar{k}_{Y}(t, s) \alpha(\mathrm{d} s) \alpha(\mathrm{d} t)=\int_{0}^{1} \int_{0}^{1} \bar{k}_{n}(t, s) \gamma(\mathrm{d} s) \gamma(\mathrm{d} t)=\|h-\bar{m}\|_{\overline{\mathcal{H}}_{n}}^{2},
$$

where $\alpha$ and $\gamma$ denote the measures representing $h-\bar{m}$ in $\mathscr{D}_{1}$ and $\mathscr{D}_{2}$, respectively, so that (A.1) is completely proved.

Now, we need to prove that, for any $h \in \mathscr{D}_{2}$, we have $\|h-\bar{m}\|_{\overline{\mathcal{H}}_{n}}^{2}=\left\|h-x_{n}\right\|_{\overline{\mathcal{H}}_{n}}^{2}-(y-$ $\left.x_{n}\right)^{2} / \bar{k}_{n}(1,1)$. This follows from the fact that $\bar{m}-x_{n}$ belongs to the reproducing kernel Hilbert space $\overline{\mathscr{H}}_{n}$, because

$$
\bar{m}_{t}-x_{n}=\frac{\bar{k}_{n}(t, 1)}{\bar{k}_{n}(1,1)}\left(y-x_{n}\right)=\int_{0}^{1} \bar{k}_{n}(t, s) \frac{y-x_{n}}{\bar{k}_{n}(1,1)} \delta_{\{1\}}(\mathrm{d} s) .
$$

Moreover, it holds that

$$
\left\|\bar{m}-x_{n}\right\|_{\overline{\mathcal{H}}_{n}}^{2}=\frac{\left(y-x_{n}\right)^{2}}{\bar{k}_{n}(1,1)} .
$$

Now take $h \in \mathcal{D}_{2}$. In particular, for some measure $\gamma$, we have $h_{t}-\bar{m}_{t}=\int_{0}^{1} \bar{k}_{n}(t, s) \gamma(\mathrm{d} s)$. Then, the measure $\hat{\gamma}(\mathrm{d} s)=\gamma(\mathrm{d} s)+\left(y-x_{n}\right) \delta_{\{1\}}(\mathrm{d} s) / \bar{k}_{n}(1,1)$ is such that $h_{t}-x_{n}=$ $\int_{0}^{1} \bar{k}_{n}(t, s) \hat{\gamma}(\mathrm{d} s)$ and

$$
\begin{aligned}
\left\langle h-x_{n}, \bar{m}-x_{n}\right\rangle_{\overline{\mathcal{H}}_{n}} & =\int_{0}^{1} \int_{0}^{1} \bar{k}_{n}(t, s)\left(\gamma(\mathrm{d} s)+\frac{y-x_{n}}{\bar{k}_{n}(1,1)} \delta_{\{1\}}(\mathrm{d} s)\right) \frac{y-x_{n}}{\bar{k}_{n}(1,1)} \delta_{\{1\}}(\mathrm{d} t) \\
& =\frac{y-x_{n}}{\bar{k}_{n}(1,1)} \int_{0}^{1} \bar{k}_{n}(1, s) \gamma(\mathrm{d} s) \\
& =\frac{y-x_{n}}{\bar{k}_{n}(1,1)}\left(h-x_{n}\right)_{1} \\
& =\frac{\left(y-x_{n}\right)^{2}}{\bar{k}_{n}(1,1)} .
\end{aligned}
$$

Therefore,

$\|h-\bar{m}\|_{\overline{\mathcal{H}}_{n}}^{2}=\left\|h-x_{n}\right\|_{\overline{\mathcal{H}}_{n}}^{2}+\left\|\bar{m}-x_{n}\right\|_{\overline{\mathcal{H}}_{n}}^{2}-2\left\langle h-x_{n}, \bar{m}-x_{n}\right\rangle_{\overline{\mathcal{H}}_{n}}=\left\|h-x_{n}\right\|_{\overline{\mathcal{H}}_{n}}^{2}-\frac{\left(y-x_{n}\right)^{2}}{\bar{k}_{n}(1,1)}$,

and the statement finally holds. 


\section{Appendix B. Proof of Proposition 4.1}

(1) Since $X$ is an integrated Gaussian process, we have

$$
\begin{aligned}
\operatorname{cov}\left(X_{T_{n}+\varepsilon t}-X_{T_{n}}, X_{T_{n}+\varepsilon s}-X_{T_{n}}\right) & =\int_{T_{n}}^{T_{n}+\varepsilon t} \mathrm{~d} u \int_{T_{n}}^{T_{n}+\varepsilon t} \mathrm{~d} v \kappa(u, v) \\
& =\varepsilon^{2} \int_{0}^{t} \mathrm{~d} u \int_{0}^{s} \mathrm{~d} v \kappa\left(T_{n}+\varepsilon u, T_{n}+\varepsilon v\right) .
\end{aligned}
$$

Therefore, we have

$$
\begin{aligned}
& \operatorname{cov}\left(X_{T_{n}+\varepsilon t}-X_{T_{n}}, X_{T_{n}+\varepsilon s}-X_{T_{n}}\right) \\
& \quad=\varepsilon^{2} \int_{0}^{t} \mathrm{~d} u \int_{0}^{s} \mathrm{~d} v\left(\kappa\left(T_{n}, T_{n}\right)+\varepsilon^{\alpha} \hat{g}(u, v)+\hat{\mathcal{R}}_{\varepsilon}(u, v)\right) \\
& \quad=\varepsilon^{2}\left(\kappa\left(T_{n}, T_{n}\right) t s+\varepsilon^{\alpha} \int_{0}^{t} \mathrm{~d} u \int_{0}^{s} \mathrm{~d} v \hat{g}(u, v)+\int_{0}^{t} \mathrm{~d} u \int_{0}^{s} \mathrm{~d} v \hat{\mathcal{R}}_{\varepsilon}(u, v)\right),
\end{aligned}
$$

so that Assumption 4.1(i) is satisfied with $\bar{\varphi}(t, s)=\int_{0}^{t} \mathrm{~d} u \int_{0}^{s} \mathrm{~d} v \hat{g}(u, v)$.

Moreover, since

$$
k\left(T_{n}+\varepsilon t, T\right)-k\left(T_{n}, T\right)=\int_{T_{n}}^{T_{n}+\varepsilon t} \mathrm{~d} u \int_{0}^{T} \mathrm{~d} v \kappa(u, v)=\varepsilon \int_{0}^{t} \mathrm{~d} u \int_{0}^{T} \mathrm{~d} v \kappa\left(T_{n}+\varepsilon u, v\right),
$$

we obtain

$$
\begin{aligned}
k\left(T_{n}+\varepsilon t, T\right)-k\left(T_{n}, T\right) & =\varepsilon\left(\int_{0}^{t} \mathrm{~d} u\left(\int_{0}^{T} \mathrm{~d} v \kappa\left(T_{n}, v\right)+\varepsilon^{\alpha} \tilde{g}(u ; T)+\tilde{\mathcal{R}}_{\varepsilon}(u ; T)\right)\right) \\
& =\varepsilon\left(t \int_{0}^{T} \mathrm{~d} v \kappa\left(T_{n}, v\right)+\varepsilon^{\alpha} \int_{0}^{t} \mathrm{~d} u \tilde{g}(u ; T)+\int_{0}^{t} \mathrm{~d} u \tilde{\mathcal{R}}_{\varepsilon}(u ; T)\right) .
\end{aligned}
$$

Then, Assumption 4.1(ii) is also satisfied with $\bar{\psi}(t, T)=\int_{0}^{t} \mathrm{~d} u \tilde{g}(u ; T)$.

The proof of part (ii) follows analogously.

\section{References}

[1] Azencott, R. (1980). Grande Déviations et Applications (École d'été de Probabilités de Saint Flour VIII; Lecture Notes Math. 774). Springer, Berlin.

[2] Baldi, P. and Caramellino, L. (2002). Asymptotics of hitting probabilities for general one-dimensional pinned diffusions. Ann. Appl. Prob. 12, 1071-1095.

[3] Baldi, P. AND PaCchiarotti, B. (2006). Explicit computation of second order moments of importance sampling estimators for fractional Brownian motion. Bernoulli 12, 663-688.

[4] Baldi, P., Caramellino, L. And Iovino, M. G. (1999). Pricing general barrier options: a numerical approach using sharp large deviations. Math. Finance 9, 293-321.

[5] Chen, X. AND Li, W. V. (2003). Quadratic functionals and small ball probabilities for the $m$-fold integrated Brownian motion. Ann. Prob. 31, 1052-1077.

[6] Cheridito, P. (2001). Mixed fractional Brownian motion. Bernoulli 7, 913-934.

[7] Dembo, A. And Zeitouni, O. (1992). Large Deviations Techniques and Applications. Jones and Bartlett, Boston, MA.

[8] Deuschel, J. D. And Stroock, D. W. (1989). Large Deviations. Academic Press, Boston, MA.

[9] Galleani, L., Sacerdote, L., Tavella, P. and Zucca, C. (2003). A mathematical model for the atomic clock error. Metrologia 40, 257-264.

[10] Gasbarra, D., Sottinen, T. and Valkeila, E. (2007). Gaussian bridges. In The Able Symposium 2005: Stochastic Analysis and Applications, eds F. Benth et al., Springer, Berlin, pp. 361-382. 
[11] Groeneboom, P., Jongbloed, G. and Wellner, J. A. (2001). A canonical process for estimation of convex functions: the 'invelope' of integrated Brownian motion $+t^{4}$. Ann. Statist. 29, 1620-1652.

[12] Mandjes, M., Mannersalo, P., Norros, I. and van Uitert, M. (2006). Large deviations of infinite intersections of events in Gaussian processes. Stoch. Process. Appl. 116, 1269-1293.

[13] Molchan, G. AND KhoKhlov, A. (2004). Small values of the maximum or the integral of fractional Brownian motion. J. Statist. Phys. 114, 923-946.

[14] Norros, I. And Saksman, A. (2007). Local independence of fractional Brownian motion. Preprint. Available at http://arXiv.org/abs/0711.4809. 